\title{
ISOTOPY TYPES OF KNOTS OF CODIMENSION TWO*
}

BY

\author{
M. S. FARBER
}

\begin{abstract}
In this paper the classification of $n$-dimensional knots in $S^{n+2}$, bounding $r$-connected manifolds, where $3 r>n+1>6$, in terms of stable homotopy theory is suggested.
\end{abstract}

The problem of isotopy classification is the fundamental one of knot theory. It was solved by A. Haefliger and J. Levine for knots of codimension $\geqslant 3$. The first step in reduction of a classification of knots of codimension two to a homotopy problem was made by R. Lashof and J. Shaneson [6]. They showed that in the class of $n$-dimensional knots with group $Z$ for $n \geqslant 5$ there are at most two different knots having homotopy equivalent exteriors. In $1970 \mathrm{~J}$. Levine [10] gave an algebraic classification of $(2 q-1)$-dimensional knots in $S^{2 q+1}$ bounding $(q-1)$ connected manifolds. It turned out that the only invariant determining the isotopy type of such knots is the Seifert matrix considered to within $S$-equivalence. Later Trotter [16] and C. Kearton [5] obtained a classification of knots, studied by $\mathrm{J}$. Levine, in terms of Blanchfield pairing. C. Kearton has partially analyzed the more difficult problem of classification of $2 q$-dimensional knots in $S^{2 q+2}$ bounding $(q-1)$-connected manifolds.

In the present paper the classification of a wider class of higher-dimensional knots is obtained. It is the class of $n$-dimensional knots in $S^{n+2}$ bounding $r$-connected manifolds, where $3 r \geqslant n+1 \geqslant 6$. The main result of this paper is the construction of a one-to-one correspondence between the set of isotopy types of such knots and some set described in purely homotopic terms.

The plan of the paper is as follows:

In $\$ 1$ submanifolds of the sphere of codimension one are considered. Such a submanifold is assigned some pairing in the sense of the Spanier-Whitehead theory. This pairing describes the homotopy linking of the submanifold with its copy translated in the direction of the positive normal field. This pairing induces the usual Seifert pairing on middle dimensional homology groups and is called the homotopy Seifert pairing. The main result of $\S 1$ is the construction of a one-to-one correspondence between the set of isotopy types of such submanifolds and the set of homotopy pairings.

Received by the editors August 7, 1979.

AMS (MOS) subject classifications (1970). Primary 57C45, 57D35; Secondary 55A25, 55D25.

Key words and phrases. $n$-knot, Seifert manifold, homotopy pairing.

* Galley proofs were read by Professor J. Levine, Department of Mathematics, Brandeis University, Waltham, Massachusetts 02154. 
$\$ 2$ deals with a situation in which nonisotopic Seifert manifolds are bounded by isotopic knots. I call this situation contiguity. In this section there is found a necessary and sufficient condition for the homotopy Seifert pairings to correspond to contiguous submanifolds.

In $\$ 3$ it is proved that two submanifolds of a sphere bound the same knot if and only if they may be connected by a sequence of contiguities. This result together with the previous theorems bring us to the classification of knots.

From these general results an algebraic classification of some classes of knots may be obtained by standard homotopy methods. This is illustrated in $\$ 3$, where the case of simple odd-dimensional knots is considered and algebraic classification in terms of Seifert matrices is obtained. This classification is essentially the same as J. Levine's [10]; the only difference is that we obtain the equivalence relation between Seifert matrices in another form. The results on algebraic classification of other classes of knots will be stated in a separate paper.

The terminology is given in the smooth category although the results of the paper are true in the piecewise linear category too.

I am grateful to A. V. Černavskiĭ for his valuable advice.

1. Classification of imbeddings of Seifert manifolds. An $\boldsymbol{n}$-dimensional knot is a pair $K=\left(S^{n+2}, k^{n}\right)$, where $S^{n+2}$ is an oriented sphere and $k^{n}$ is its oriented $n$-dimensional submanifold which is a homotopy sphere. A Seifert manifold of a knot $K$ is any compact connected oriented $(n+1)$-dimensional submanifold $V \subset$ $S^{n+2}$ with $\partial V=k$. In this section it is proved that the isotopy type of $V$ is determined by the homotopy type of $V$ and some homotopy pairing. Here also necessary and sufficient conditions are determined under which the finite complex with the given homotopy pairing may be realized by the $(n+1)$-dimensional submanifold $V \subset S^{n+2}$ for which $\partial V$ is the homotopy sphere.

1.1. Let $V$ be a connected oriented $(n+1)$-dimensional submanifold of the oriented sphere $S^{n+2}$. Suppose that the boundary $\partial V$ is nonempty. Let $Y$ be the closure of the complement of a tubular neighborhood of $V$ in $S^{n+2}$. Let $u$ : $V \wedge Y \rightarrow S^{n+1}$ be the canonical pairing (see, for example, [15, Chapter 3]) which is the Spanier-Whitehead duality [13]. Denote by $i_{+}: V \rightarrow Y$ the mapping which is given by small translation along the field of positive normals to $V$. By homotopy Seifert pairing of $V$ we shall call the composition

$$
\theta: V \wedge V^{1 \wedge i_{+}} \rightarrow \stackrel{u}{\rightarrow} V \wedge \stackrel{u}{\rightarrow} S^{n+1}
$$

It is clear that $\theta$ is determined by imbedding $V \subset S^{n+2}$ uniquely up to homotopy.

If $n=2 q-1$, then homotopy pairing $\theta$ defines homology pairing $H_{q}(V) \otimes$ $H_{q}(V) \rightarrow \mathbf{Z}$ by the formula $z_{1} \otimes z_{2} \mapsto \theta_{*}\left(z_{1} \wedge z_{2}\right) \in H_{2 q}\left(S^{2 q}\right)=\mathbf{Z}$. This homology pairing coincides with Seifert pairing [9], [12].

1.2. TheOREM. Let $V_{1}$ and $V_{2}$ be two compact oriented $(n+1)$-dimensional submanifolds of sphere $S^{n+2}$ with $\partial V_{1}$ and $\partial V_{2}$ homotopy spheres. Let $\theta_{i}: V_{i} \wedge V_{i} \rightarrow$ $S^{n+1}, i=1,2$, be corresponding homotopy Seifert pairings. Suppose that the manifolds $V_{1}$ and $V_{2}$ are $r$-connected, where $3 r \geqslant n+1, n \geqslant 5$. If there is a homotopy 
equivalence $f: V_{1} \rightarrow V_{2}$ for which $\theta_{2} \circ(f \wedge f)$ is homotopic to $\theta_{1}$, then there exists isotopy of sphere $S^{n+2}$ which transfers $V_{1}$ on $V_{2}$ with preservation of orientations.

1.3. THEOREM. Let $K$ be a finite r-connected $k$-dimensional complex and $\theta$ : $K \wedge K \rightarrow S^{n+1}$ be a continuous map. If $2 n>3 k, 2 k \leqslant n+r, n>4, r \geqslant 1$, then there exists an $(n+1)$-dimensional oriented submanifold $V \subset S^{n+2}$ with $\pi_{1}(\partial V)=1$ and homotopy equivalence $g: V \rightarrow K$ such that $\theta \circ(g \wedge g): V \wedge V \rightarrow S^{n+1}$ is homotopic to the homotopy Seifert pairing of $V$.

The proofs of Theorems 1.2 and 1.3 will be given at the end of this section.

When we shall apply Theorem 1.3 it will be important to know under which conditions the homotopy pairing $\theta: K \wedge K \rightarrow S^{n+1}$ is realized by submanifold $V^{n+1}$ for which $\partial V$ is the homotopy sphere. The following theorem answers this question.

1.4. THEOREM. Let $V$ be a connected $(n+1)$-dimensional oriented submanifold of sphere $S^{n+2}$ with $\partial V \neq \varnothing$, and let $\theta: V \wedge V \rightarrow S^{n+1}$ be its homotopy Seifert pairing. The boundary $\partial V$ is a homology sphere if and only if the following pairing

$$
\theta+(-1)^{n+1} \theta^{\prime}: V \wedge V \rightarrow S^{n+1}
$$

is the Spanier-Whitehead duality. Here $\theta^{\prime}$ is the composition of the map $T: V \wedge V \rightarrow$ $V \wedge V$, which transposes the coordinates, and $\theta$; the signs plus and minus are understood as operations in the cohomotopy group $\pi^{n+1}(V \wedge V)$.

REMARK. This cohomotopy group exists since $H^{i}(V \wedge V)=0$ for $i>2 n$.

Proof of TheOREM 1.4. Let $Y$ be a complement of an open tubular neighborhood of $V$ in $S^{n+2}$ and let $i_{+}: V \rightarrow Y$ and $i_{-}: V \rightarrow Y$ be given by translations in the directions of positive and negative normals to $V$, respectively.

Let us first prove that $\partial V$ is the homology sphere if and only if the homomorphisms

$$
i_{+*}-i_{-*}: H_{k} V \rightarrow H_{k} Y
$$

are isomorphisms for $k>0$. Consider the isomorphism

$$
\Psi: H_{k} Y \rightarrow H_{k}(V, \partial V), \quad 0<k<n+1,
$$

which is the composition

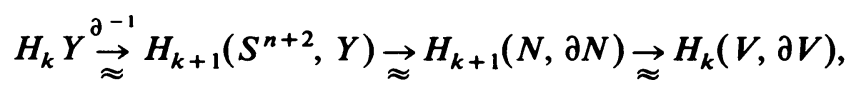

where the first homomorphism is reverse to boundary homomorphism, the second is an isomorphism of excision (here $N$ is the tubular neighborhood of $V$ in $S^{n+2}$ ) and the third is an isomorphism which exists by virtue of $(N, \partial N)=(V, \partial V) \times$ $(I, \partial I)$.

The composition

$$
H_{k} V^{i_{+*}-i_{-*}} H_{k} Y \stackrel{\Psi}{\rightarrow} H_{k}(V, \partial V)
$$

coincides with the homomorphism induced by inclusion $V \rightarrow(V, \partial V)$. Since $\Psi$ is an isomorphism, then $i_{+*}-i_{-*}$ is an isomorphism if and only if the inclusion 
$V \rightarrow(V, \partial V)$ induces an isomorphism of $k$-dimensional homologies for $0<k<n$

+1 . But this is equivalent to $\partial V$ being a homology sphere.

Consider the following diagram:

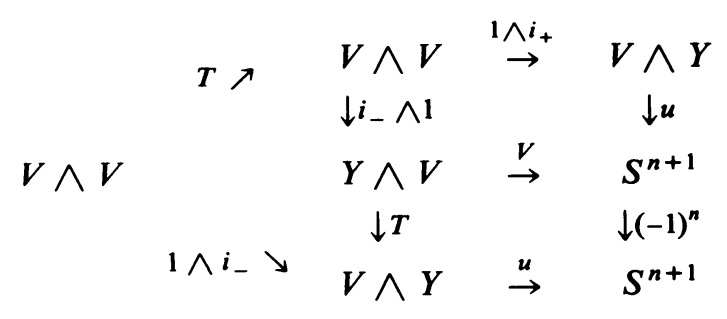

Here $u$ and $v$ are canonical pairings which are Spanier-Whitehead dualities and $(-1)^{n}$ denotes the map of degree $(-1)^{n}$. This diagram is homotopically commutative. Therefore $\theta^{\prime}=\theta \circ T=u \circ\left(1 \wedge i_{+}\right) \circ T \sim(-1)^{n} u \circ\left(1 \wedge i_{-}\right)$. If $s \in H^{n+1} S^{n+1}$ is a generator and $z \in H_{k} V$, then

$$
\begin{aligned}
{\left[\theta+(-1)^{n+1} \theta^{\prime}\right]^{*} s / z } & =\theta^{*} s / z+(-1)^{n+1} \theta^{* *} s / z \\
& =\left(1 \wedge i_{+}\right)^{*} \circ u^{*} s / z-\left(1 \wedge i_{-}\right)^{*} \circ u^{*} s / z \\
& =u^{*} s / i_{+*} z-u^{*} s / i_{-*} z=u^{*} s /\left(i_{+*}-i_{-*}\right) z .
\end{aligned}
$$

Thus $\left[\theta+(-1)^{n+1} \theta^{\prime}\right]^{*} s / z=u^{*} s /\left(i_{+*}-i_{-*}\right) z$. Since $u$ is a Spanier-Whitehead duality, then it follows from this formula that the map $H_{k} V \rightarrow H^{n+1-k} V, 0<k<$ $n+1$ given by the formula

$$
z \mapsto\left[\theta+(-1)^{n+1} \theta^{\prime}\right]^{*} s / z
$$

is an isomorphism if and only if $i_{+*}-i_{-*}$ is an isomorphism.

This proves Theorem 1.4.

1.5. For the proofs of Theorems 1.2 and 1.3 some facts of the thickening theory [17] are needed.

Let $K^{k}$ be a finite connected $\mathrm{CW}$-complex of dimension $k$ with base point *. Let $M^{m}$ be a compact manifold with base point $* \in \partial M$ and fixed orientation of the tangent space to $M$ in the point *. Suppose that $m \geqslant k+3$ and the inclusion $i$ : $\partial M \rightarrow M$ induces an isomorphism $\pi_{1}(\partial M) \rightarrow \pi_{1}(M)$. The simple homotopy equivalence $\varphi:(K, *) \rightarrow(M, *)$ is called the $m$-dimensional thickening of $K$. Two $m$-dimensional thickenings $\varphi_{1}:(K, *) \rightarrow\left(M_{1}, *\right)$ and $\varphi_{2}:(K, *) \rightarrow\left(M_{2}, *\right)$ are called equivalent if there is a diffeomorphism $h: M_{1} \rightarrow M_{2}$ preserving the base points and the orientation in them, and such that

$$
h \circ \varphi_{1} \sim \varphi_{2}:(K, *) \rightarrow\left(M_{2}, *\right) .
$$

1.6. THEOREM. Let $M^{m}$ be a manifold, $K^{k}$ be a finite $C W$-complex, $f: K \rightarrow M$ be some $(2 k-m+1)$-connected map and $k \leqslant m-3$. Then there exist a compact submanifold $N^{m}$ of $M^{m}$ with $\pi_{1}(\partial N)=\pi_{1}(N)$ and a simple homotopy equivalence $g$ : $K \rightarrow N$ such that $g \sim f: K \rightarrow M$ and this homotopy preserves base points. 
1.7. Theorem. Let $f_{1}: K \rightarrow N_{1}^{m}$ and $f_{2}: K \rightarrow N_{2}^{m}$ be two simple homotopy equivalences where $N_{1}$ and $N_{2}$ are submanifolds of manifold $M^{m}$ with $\pi_{1}\left(\partial N_{1}\right)=\pi_{1}\left(N_{1}\right)$, $\pi_{1}\left(\partial N_{2}\right)=\pi_{1}\left(N_{2}\right)$ and $k=\operatorname{dim} K \leqslant m-3, m \geqslant 6$. If $f_{1}$ and $f_{2}$ are homotopic in $M$ rel$^{*}$ and the compositions

$$
K \stackrel{f_{v}}{\rightarrow} N_{\nu} \stackrel{\ulcorner}{\rightarrow} M, \quad \nu=1,2,
$$

are $(2 k-m+2)$-connected, then there exists an isotopy $h_{t}: M \rightarrow M$ such that $h_{0}=\mathrm{id}, h_{t}=(*)=1, h_{1}\left(N_{1}\right)=N_{2}$ and the diagram

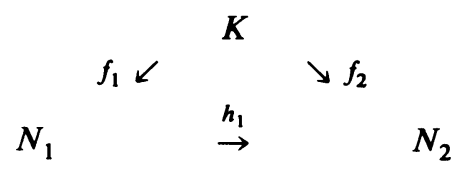

is homotopy commutative.

Theorem 1.6 is the first part of Wall's Embedding theorem [17, p. 76]. Theorem 1.7 makes precise the second part of the Wall theorem with regard to the results of [3], [11].

The simple homotopy equivalence $g: K \rightarrow N$ which existence is affirmed in Theorem 1.6 is called the thickening induced by the map $f: K \rightarrow M$. Theorem 1.7 means uniqueness of induced thickening.

For the proofs of Theorems 1.2 and 1.3 we also need to use the next statement.

1.8. Proposition. Let $N^{n+2}$ be a compact submanifold of sphere $S^{n+2}$ having the homotopy type of a finite $k$-dimensional $r$-connected $C W$-complex, where $r \geqslant 1$, $n \geqslant 4, n>k$. Let $Y=\operatorname{cl}\left(S^{n+2}-N\right)$. The inclusions $i: \partial N \rightarrow N$ and $j: \partial N \rightarrow Y$ determine the map $\psi: \partial N \rightarrow N \times Y, \psi(x)=(i(x), j(x))$. The map $\psi_{\#}:[L, \partial N] \rightarrow$ $[L, N] \times[L, Y]$ induced by $\psi$ is surjective if $\operatorname{dim} L \leqslant n+r-k+1$ and bijective if $\operatorname{dim} L \leqslant n+r-k$.

Proof. It is enough to show that the spaces $N, Y, \partial N$ are simply connected and the map $\psi$ induces isomorphism of homology groups in dimensions $<n+r-k$ +1 . $N$ is simply connected by hypotheses. Since $n+2 \geqslant 6$, then $N$ has a handle decomposition with handles of index $\leqslant k$. The dual decomposition begins on $\partial N \times[0,1]$ and has the handles of index $>n+2-k>3$. Therefore $\pi_{1}(\partial N)=$ $\pi_{1}(N)=1$. It follows now from Van Kampen's theorem that $\pi_{1}(Y)=1$.

Notice that $Y$ is $(n-k)$-connected since $H_{s} Y=H^{n+1-s} N=0$, if $s<n-k$. Therefore any class $z^{\prime} \in H_{s}(N \times Y)$ for $s<(n-k+1)+(r+1)$ is uniquely presented as $z^{\prime}=z_{1} \times 1+1 \times z_{2}$, where $z_{1} \in H_{s} N$ and $z_{2} \in H_{s} Y$. If $z \in H_{s}(\partial N)$, then it is clear that $\psi_{*} z=i_{*} z \times 1+1 \times j_{*} z$. It follows from the Mayer-Vietoris sequence of the pair $\{N, Y\}$ that we have isomorphism

$$
\tilde{H}_{s}(\partial N) \stackrel{i * \otimes_{j *}}{\rightarrow} \tilde{H}_{s} N \oplus \tilde{H}_{s} T
$$

Consequently $\psi_{*}: H_{s}(\partial N) \rightarrow H_{s}(N \times Y)$ is an isomorphism for $s<n+r-k+$ 1.

The proposition is proved. 
1.9. Proof of Theorem 1.2. Denote $N_{\nu}=V_{\nu} \times[0,1], \nu=1$, 2. Identify $V_{\nu}$ with $V_{\nu} \times 0 \subset N_{\nu}$ and denote by $i_{\nu}$ the corresponding inclusion $V_{\nu} \rightarrow N_{\nu}, \nu=1,2$. The imbedding of $V_{\nu}$ in $S^{n+2}$ may be extended to imbedding $N_{\nu} \rightarrow S^{n+2}$ such that for each point $x \in V_{\nu}$ the curve $\{(x, t) ; t \in[0,1]\}$ goes out from $x=(x, 0)$ along the direction of the negative normal to $V_{\nu}, \nu=1,2$.

Manifolds $V_{\nu}$ are simply connected and for $s>n-r$ we have $H^{s} V_{\nu}=$ $H_{n+1-s}\left(V_{v}, \partial V_{v}\right)=\tilde{H}_{n+1-s} V_{\nu}=0, \nu=1,2$. Here we have used that $\partial V_{\nu}$ is a homotopy sphere. Thus there exists a finite CW-complex $K$ of dimension $<n-r$ and homotopy equivalence $\varphi_{1}: K \rightarrow V_{1}$. Denote the composition $\varphi_{2}=f \circ \varphi_{1}$ by $\varphi_{2}$ : $K \rightarrow V_{2}$.

The maps $i_{1} \circ \varphi_{1}: K \rightarrow N_{1}$ and $i_{2} \circ \varphi_{2}: K \rightarrow N_{2}$ are $(n+2)$-dimensional thickenings of complex $K$. According to Theorem 1.7 (here all conditions of this theorem are satisfied) there exists isotopy $h_{t}$ of sphere $S^{n+2}$ such that $h_{0}=\mathrm{id}, h_{1}\left(N_{1}\right)=N_{2}$ and $h_{1} \circ i_{1} \circ \varphi_{1}$ is homotopic to $i_{2} \circ \varphi_{2}$ in $N_{2}$.

I want to prove that manifolds $V_{2}$ and $h_{1}\left(V_{1}\right)$ are isotopic on $\partial N_{2}$. Since $V_{2}$ is the thickening induced by the map

$$
\alpha_{2}: K \stackrel{\varphi_{2}}{\rightarrow} V_{2} \stackrel{\ulcorner}{\rightarrow} \partial N_{2},
$$

and $h_{1}\left(V_{1}\right)$ is the thickening induced by the map

$$
\alpha_{1}: K \stackrel{\varphi_{1}}{\rightarrow} V_{1} \stackrel{h_{1}}{\rightarrow} h_{1}\left(V_{1}\right) \stackrel{\subsetneq}{\rightarrow} \partial N_{2}
$$

then by virtue of Theorem 1.7 it is enough to show that each of the maps $\alpha_{1}$ and $\alpha_{2}$ are $2(n-r)-(n+1)+2$-connected and $\alpha_{1} \sim \alpha_{2}: K \rightarrow \partial N_{2}$. The connectivity conditions for $\alpha_{1}$ and $\alpha_{2}$ are obviously satisfied since $3 r>n+1$.

Let us show that $\alpha_{1} \sim \alpha_{2}$. By virtue of Proposition 1.8 it is enough to prove that

(1) $i \circ \alpha_{1} \sim i \circ \alpha_{2}$ in $N_{2}$,

(2) $j \circ \alpha_{1} \sim j \circ \alpha_{2}$ in $Y$,

where $i: \partial N_{2} \rightarrow N_{2}$ and $j: \partial N_{2} \rightarrow Y$ are inclusions and $Y=S^{n+2}-$ int $N_{2}$. The relation (1) can be easily verified:

$$
i \circ \alpha_{2}=i_{2} \circ \varphi_{2} \sim h_{1} \circ i_{1} \circ \varphi_{1}=i \circ \alpha_{1} \text {. }
$$

Let us verify the relation (2). Let $N$ be obtained from $N_{2}$ by removing some collar of the boundary $\partial N_{2}$. By $r: N_{2} \rightarrow \partial N$ we denote a natural retraction and by $u$ : $N \wedge Y \rightarrow S^{n+1}$ a canonical pairing which is a Spanier-Whitehead duality. It is clear that the composition $V_{2} \wedge V_{2} \stackrel{i \wedge j}{\rightarrow} N_{2} \wedge Y \stackrel{r \wedge 1}{\rightarrow} N \wedge Y \stackrel{u}{\rightarrow} S^{n+1}$ is homotopic to homotopy Seifert pairing $\theta_{2}$ of manifold $V_{2}$. Analogously the composition

$$
V_{1} \wedge V_{1} \stackrel{h_{1} \wedge h_{1}}{\rightarrow} h_{1}\left(V_{1}\right) \wedge h_{1}\left(V_{1}\right) \stackrel{i \wedge j}{\rightarrow} N_{2} \wedge Y^{r \wedge 1} \rightarrow N \wedge Y \stackrel{u}{\rightarrow} S^{n+1}
$$

is homotopic to $\theta_{1}$. Therefore

$$
\begin{aligned}
& \theta_{2} \circ\left(\varphi_{2} \wedge \varphi_{2}\right) \sim u \circ\left(r \circ i \circ \varphi_{2} \wedge j \circ \varphi_{2}\right), \\
& \theta_{1} \circ\left(\varphi_{1} \wedge \varphi_{1}\right) \sim u \circ\left(r \circ i \circ h_{1} \circ \varphi_{1} \wedge j \circ h_{1} \circ \varphi_{1}\right) .
\end{aligned}
$$

On the other hand, since $\varphi_{2}=f \circ \varphi_{1}$, then

$$
\theta_{2} \circ\left(\varphi_{2} \wedge \varphi_{2}\right)=\theta \circ(f \wedge f) \circ\left(\varphi_{1} \wedge \varphi_{1}\right) \sim \theta_{1} \circ\left(\varphi_{1} \wedge \varphi_{1}\right) .
$$


Thus

$$
u \circ\left(r \circ i \circ \varphi_{2} \wedge j \circ \varphi_{2}\right) \sim u \circ\left(r \circ i \circ h_{1} \circ \varphi_{1} \wedge j \circ h_{1} \circ \varphi_{1}\right) .
$$

We have

$$
\begin{aligned}
& i \circ \varphi_{2}=i \circ \alpha_{1} \sim i \circ \alpha_{2}=i \circ h_{1} \circ \varphi_{1}, \\
& j \circ \varphi_{2}=j \circ \alpha_{2}, \quad j \circ h_{1} \circ \varphi_{1}=j \circ \alpha_{1} .
\end{aligned}
$$

Consequently

$$
u \circ\left(\beta \wedge j \circ \alpha_{2}\right) \sim u\left(\beta \wedge j \circ \alpha_{1}\right), \text { where } \beta=r \circ i \circ \varphi_{2} \sim r \circ i \circ h_{1} \circ \varphi_{1} .
$$

It follows from the last relation that $j \circ \alpha_{2} \sim j \circ \alpha_{1}$. In fact, since $u$ is a SpanierWhitehead duality and $\beta$ is homotopy equivalent, then the map $\{K, Y\} \rightarrow\{K \wedge$ $\left.K, S^{n+1}\right\}$, which transfers the class of $S$-map $\gamma: K \rightarrow Y$ into the class of $u \circ(\beta \wedge$ $\gamma$ ), is isomorphism. It means that $j \circ \alpha_{2}$ is stably homotopic to $j \circ \alpha_{1}$. On the other hand $[K, Y]=\{K, Y\}$ since $Y$ is $r$-connected and $\operatorname{dim} K<n-r$.

Thus $\alpha_{1}$ is homotopic to $\alpha_{2}$ on $\partial N_{2}$ and so the isotopy of $\partial N_{2}$ exists which translates $V_{2}$ on $h_{1}\left(V_{1}\right)$ with preservation of orientations. This isotopy may be extended to the isotopy of sphere $S^{n+2}$.

The theorem is proved.

1.10. Proof of Theorem 1.3. Let $S^{n+1}$ be the equator of sphere $S^{n+2}$. Since $r \geqslant 2 k-n$, then by virtue of Theorem 1.6 an $(n+1)$-dimensional trivial thickening of complex $K$ exists, i.e. submanifold $N_{0}^{n+1} \subset S^{n+1}$ and homotopy equivalence $\varphi: K \rightarrow N_{0}$. Fix some orientation on $N_{0}$. Let us identify manifold $N_{0}$ with $N_{0} \times 0$ $\subset N_{0} \times[0,1]=N$. Imbed manifold $N$ in $S^{n+2}$ so that this imbedding will be the extension of the imbedding $N_{0} \subset S^{n+1}$ and so that for each point $x \in N_{0}$ the curve $\{(x, t) ; t \in[0,1]\}$ goes out from $x$ along the direction of negative normal to $N_{0}$ in $S^{n+2}$.

Let $Y$ be a closure of complement to $N$ in sphere $S^{n+2}$ and let $Y^{\prime}$ be obtained from $Y$ by removing some small collar of boundary $\partial Y$. By $v: N \wedge Y^{\prime} \rightarrow S^{n+1}$ denote the canonical Spanier-Whitehead duality and by $r: Y \rightarrow Y^{\prime}$ the natural retraction. The map $u: N \wedge Y \stackrel{1 \wedge r}{\rightarrow} N \wedge Y^{\prime} \stackrel{v}{\rightarrow} S^{n+1}$ is also a Spanier-Whitehead duality. By virtue of Spanier-Whitehead theory there exists a unique element $\{h\} \in\{K, Y\}$ such that the composition

$$
K \wedge K \stackrel{\alpha \wedge h}{\rightarrow} N \wedge Y \stackrel{u}{\rightarrow} S^{n+1}
$$

is stably homotopic to $\theta$, where $\alpha: K \rightarrow N$ is the composition of homotopy equivalence $\varphi: K \rightarrow N_{0}$ and imbedding $N_{0} \rightarrow N$. Note that $Y$ is $(n-k)$-connected and since $3 k \leqslant 2 n$, then $\{K, Y\}=[K, Y]$. Besides $\left\{K \wedge K, S^{n+1}\right\}=[K \wedge$ $K, S^{n+1}$ ] since $k<n$. Thus we can regard that $h: K \rightarrow Y$ is the mapping and $\theta \sim u \circ(\alpha \wedge h)$.

By virtue of Proposition 1.8 there is the map $e: K \rightarrow \partial N$ with $\psi_{\#}([e])=([\alpha],[h])$, where $\psi:[K, \partial N] \rightarrow[K, N] \times[K, Y]$. We want to apply Theorem 1.6 to the mapping $e: K \rightarrow \partial N$. The manifold $\partial N$ is $\min \{r, n-k\}$-connected and since $2 k-(n+1)+1 \leqslant \min \{r, n-k\}$, then in this case Theorem 1.6 is applicable. Let $f: K \rightarrow V^{n+1}$ be the thickening induced by the mapping $e: K \rightarrow \partial N$. Here $V^{n+1}$ is the submanifold of $\partial N$ with $\pi_{1}(\partial V)=\pi_{1}(V)=1$ and $f$ is homotopy equivalent. 
Orient $V$ so that $N_{0}$ and $V$ define the same orientation of $\partial N$.

Let $\beta: V \rightarrow \partial N$ be an inclusion. Homotopy Seifert pairing of $V$ is homotopic to the composition

$$
V \wedge V^{\beta} \stackrel{\beta}{\rightarrow} \partial N \wedge \partial \stackrel{i \wedge j}{\rightarrow} N \wedge Y \stackrel{u}{\rightarrow} S^{n+1}
$$

where $i: \partial N \rightarrow N, j: \partial N \rightarrow Y$ are inclusions. According to the construction

$$
\beta \circ f \sim e, \quad i \circ e \sim \alpha, \quad j \circ e \sim h, \quad u \circ(\alpha \wedge h) \sim \theta .
$$

Thus the following diagram

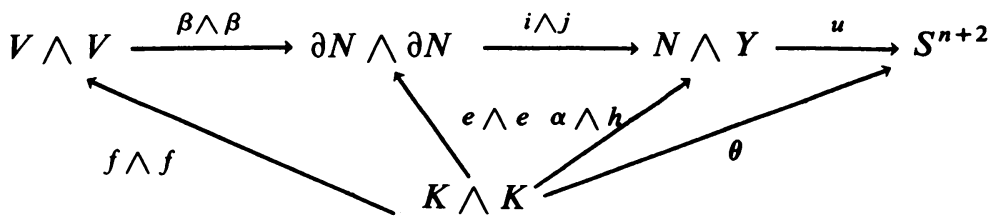

is homotopy commutative. Since the composition of the horizontal line of this diagram is homotopic to the homotopy Seifert pairing of $V$, then the latter is homotopic to $\theta \circ(g \wedge g)$, where $g$ is the homotopy equivalent inverse to $f$.

The theorem is proved.

1.11. By $M_{r, n}$ we denote the set of isotopy classes of $r$-connected $(n+1)$ dimensional oriented submanifolds of sphere $S^{n+2}$ bounding the homotopy spheres.

Consider also the set of pairs $(K, \theta)$, where $K$ is a finite $r$-connected complex and and $\theta: K \wedge K \rightarrow S^{n+1}$ is a homotopy pairing for which $\theta+(-1)^{n+1} \theta^{\prime}$ is the Spanier-Whitehead duality. We shall call two such pairs $\left(K_{i}, \theta_{i}\right), i=1,2$, equivalent if there is homotopy equivalence $f: K_{1} \rightarrow K_{2}$ such that $\theta_{1}$ is homotopic to $\theta_{2} \circ(f \wedge f)$. We denote the set of equivalence classes by $Z_{r, n}$.

The next statement follows from Theorems 1.2, 1.3 and 1.4.

1.12. Corollary. If $3 r \geqslant n+1 \geqslant 6$, then the map $M_{r, n} \rightarrow Z_{r, n}$ which assigns to the submanifold of codimension one of sphere $S^{n+2}$ its homotopy Seifert pairing is bijective.

Actually, by virtue of Theorem 1.2 this map is injective and according to Theorem 1.4 its values belong to $Z_{r, n}$. Surjectivity of this map follows from Theorem 1.3 (it is necessary to note that if $(K, \theta)$ is some element of $Z_{r, n}$, then duality $K \wedge K \rightarrow S^{n+1}$ exists and thus $K$ has a homotopy type $(n-r)$-connected complex) and Theorem 1.4.

2. Contiguity. In this section the simplest situation is considered when nonisotopic $(n+1)$-dimensional submanifolds of sphere $S^{n+2}$ bound isotopic knots, and relations are found between their homotopy Seifert pairings.

2.1. Proposition. Let $N$ be a connected $(n+2)$-dimensional compact submanifold of sphere $S^{n+2}$ and let $D^{n+1}$ be a small disk lying on $\partial N$. Denote $V=\partial N-$ int $D^{n+1}, Y=S^{n+2}-$ int $N$. Let $i: V \rightarrow N$ and $j: V \rightarrow Y$ be inclusions. Then there exists an $S$-map $f: N \vee Y \rightarrow V$ which is stable homotopy equivalent and such that 


$$
i \circ f \circ k \stackrel{s}{\sim} 1_{N}, \quad i \circ f \circ l \stackrel{s}{\sim} 0, \quad j \circ f \circ k \stackrel{s}{\sim} 0, \quad j \circ f \circ l \stackrel{s}{\sim} 1_{Y}
$$

where $k: N \rightarrow N \vee Y$ and $l: Y \rightarrow N \vee Y$ are inclusions; sign $\stackrel{s}{\sim}$ means "stably homotopic".

Proof. Let manifold $N_{1}$ be obtained from $N$ by removing some small collar of the boundary of $N$. Analogously, $Y_{1}$ is obtained from $Y$ by removing the small collar of $\partial Y$. By $r: N \rightarrow N_{1}$ and $s: Y \rightarrow Y_{1}$, we denote the natural retractions. Since $N_{1}$ and $Y_{1}$ are disjoint subsets of sphere $S^{n+2}$, then the canonical pairings

$$
v: N_{1} \wedge Y_{1} \rightarrow S^{n+1}, \quad u: Y_{1} \wedge N_{1} \rightarrow S^{n+1}
$$

are defined. Since $N_{1}$ and $Y_{1}$ are complementary to each other, then $u$ and $v$ are Spanier-Whitehead dualities.

Let $\omega$ be a simple smooth arc in sphere $S^{n+2}$ which does not intersect $V U$ int $N_{1} \cup$ int $Y_{1}$ and such that its origin lies on $\partial N_{1}$ and its end lies on $\partial Y_{1}$. Suppose that $\omega$ transversally intersects $\partial N_{1}$ and $\partial Y_{1}$. Obviously, the space $Z=N_{1} \cup Y_{1} \cup \omega$ is a deformation retract of complement $S^{n+2}-V$ and therefore the canonical pairing

$$
w: V \wedge Z \rightarrow S^{n+1}
$$

is a Spanier-Whitehead duality. If $i_{1}: N_{1} \rightarrow Z$ and $i_{2}: Y_{1} \rightarrow Z$ are inclusions, then

$$
\begin{aligned}
& w \circ\left(1_{V} \wedge i_{1}\right) \stackrel{s}{\sim} u \circ\left(s \circ j \wedge 1_{N_{1}}\right) \\
& w \circ\left(1_{Y} \wedge i_{2}\right) \stackrel{s}{\sim} v \circ\left(r \circ i \wedge 1_{Y_{1}}\right)
\end{aligned}
$$

Let the map $M: Z \wedge Z \rightarrow S^{n+1}$ be given by the matrix $\left(\begin{array}{ll}0 & v \\ u & 0\end{array}\right)$, i.e.

$$
\begin{array}{ll}
M \circ\left(i_{1} \wedge i_{1}\right)=0, & M \circ\left(i_{1} \wedge i_{2}\right)=v, \\
M \circ\left(i_{2} \wedge i_{1}\right)=u, & M \circ\left(i_{2} \wedge i_{2}\right)=0 .
\end{array}
$$

It is easy to verify that $M$ is a Spanier-Whitehead duality. Since $M$ and $w$ are the dualities, then by virtue of Spanier-Whitehead theory there exists an $S$-map $h$ : $Z \rightarrow V$ which is stable homotopy equivalent such that

$$
w \circ\left(h \wedge 1_{z}\right) \stackrel{s}{\sim} M
$$

Let us prove that

$$
\begin{aligned}
& r \circ i \circ h \circ i_{1} \stackrel{s}{\sim} 1_{N_{1}}, \\
& r \circ i \circ h \circ i_{2} \stackrel{s}{\sim} 0, \\
& s \circ j \circ h \circ i_{1} \stackrel{s}{\sim} 0, \\
& s \circ j \circ h \circ i_{2} \stackrel{s}{\sim} 1_{Y_{1}}
\end{aligned}
$$


We have

$$
\begin{aligned}
v \circ\left(r \circ i \circ h \circ i_{1} \wedge 1_{Y_{1}}\right) & \\
= & v \circ\left(r \circ i \wedge 1_{Y_{1}}\right) \circ\left(h \circ i_{1} \wedge 1_{Y_{1}}\right) \stackrel{s}{\sim} w \circ\left(1_{v} \wedge i_{2}\right) \circ\left(h \circ i_{1} \wedge 1_{Y_{1}}\right) \\
& =w \circ\left(h \wedge 1_{z}\right) \circ\left(i_{1} \wedge i_{2}\right) \stackrel{s}{\sim} M \circ\left(i_{1} \wedge i_{2}\right)=v .
\end{aligned}
$$

Here homotopies (2), (4) and (3) were used. Thus $v \circ\left(r \circ i \circ h \circ i_{1} \wedge 1_{Y_{1}}\right) \stackrel{s}{\sim} v$. It follows now that $r \circ i \circ h \circ i_{1} \stackrel{S}{\sim} 1_{N_{1}}$ since $v$ is a Spanier-Whitehead duality. Thus (5) is proved.

For the proof of (8) we have

$$
\begin{aligned}
u \circ\left(s \circ j \circ h \circ i_{2} \wedge 1_{N_{1}}\right) & \\
= & u \circ\left(s \circ j \wedge 1_{N_{1}}\right) \circ\left(h \circ i_{2} \wedge 1_{N_{1}}\right){\stackrel{s}{\sim} w \circ\left(1_{v} \wedge i_{1}\right) \circ\left(h \circ i_{2} \wedge 1_{N_{1}}\right)}=w \circ\left(h \wedge 1_{z}\right) \circ\left(i_{2} \wedge i_{1}\right) \stackrel{s}{\sim} M \circ\left(i_{2} \wedge i_{1}\right)=u .
\end{aligned}
$$

From this it follows that $s \circ j \circ h \circ i_{2} \stackrel{s}{\sim} 1_{Y_{1}}$ as above. The relations (6) and (7) may be proved analogously.

Let the map $g: Z \rightarrow N_{1} \vee Y_{1}$ be as follows. $g$ identically maps $N_{1}$ and $Y_{1}$ and maps the arc $\omega$ in the base point of $N_{1} \vee Y_{1}$. Consider the composition

$$
f: N \vee Y \stackrel{r \vee s}{\rightarrow} N_{1} \vee Y_{1} \rightarrow Z \stackrel{h}{\rightarrow} V
$$

of the map $r \vee s$, homotopy equivalence, which is inverse to $g$, and the map $h$. It follows from relations (5), (6), (7), (8) that $f$ satisfies all required conditions.

The proposition is proved.

2.2. Corollary. Let $N$ be a connected $(n+2)$-dimensional compact submanifold of sphere $S^{n+2}$ and let $D^{n+1}$ be a small disk on $\partial N$. Let $V=\partial N-\operatorname{int} D^{n+1}$, $Y=S^{n+2}-$ int $N$. Suppose that the manifold $V$ is oriented so that the field of positive normals to $V$ is directed to the exterior of $N$. Then there exists an $S$-map $f$ : $N \vee Y \rightarrow V$ which is stable homotopy equivalent and such that the map

$$
\theta \circ(f \wedge f):(N \vee Y) \wedge(N \vee Y) \rightarrow S^{n+1}
$$

is given by the matrix $\left(\begin{array}{ll}0 & u \\ 0 & 0\end{array}\right)$ (this means that

$$
\begin{array}{cl}
\theta \circ(f \wedge f) \circ(k \wedge k) \sim 0, & \theta \circ(f \wedge f) \circ(k \wedge l) \sim u, \\
\theta \circ(f \wedge f) \circ(l \wedge k) \sim 0, & \theta \circ(f \wedge f) \circ(l \wedge l) \sim 0,
\end{array}
$$

where $k: N \rightarrow N \vee Y$ and $l: Y \rightarrow N \vee Y$ are inclusions), where $\theta: V \wedge V \rightarrow S^{n+1}$ is the homotopy Siefert pairing of $V$ and $u$ is a Spanier-Whitehead duality.

Proof. It follows from the definition that the homotopy pairing $\theta$ is homotopic to the composition

$$
V \wedge V \stackrel{i \wedge j}{\rightarrow} N \wedge Y^{v \circ(r \wedge s)} S^{n+1}
$$

where the notations are the same as in the proof of Proposition 2.1. Let $f$ : $N \vee Y \rightarrow V$ be the stable homotopy equivalence constructed in Proposition 2.1. 
Then

$$
\theta \circ(f \wedge f) \sim v \circ(r \wedge s) \circ(i \wedge j) \circ(f \wedge f)=v \circ(r \wedge s) \circ(i \circ f \wedge j \circ f) .
$$

Therefore

$$
\begin{aligned}
\theta \circ(f \wedge f) \circ(k \wedge k) & \sim v \circ(r \wedge s) \circ(i \circ f \circ k \wedge j \circ f \circ k) \\
& \sim v \circ(r \wedge s) \circ\left(1_{N} \wedge 0\right)=0,
\end{aligned}
$$

and

$$
\begin{aligned}
\theta \circ(f \wedge f) \circ(k \wedge l) & \sim v \circ(r \wedge s) \circ(i \circ f \circ k \wedge j \circ f \circ l) \\
& \sim v \circ(r \wedge s) \circ\left(1_{N} \wedge 1_{Y}\right)=v \circ(r \wedge s)=u
\end{aligned}
$$

is a Spanier-Whitehead duality. It may be proved analogously that

$$
\theta \circ(f \wedge f) \circ(l \wedge k) \sim 0, \quad \theta \circ(f \wedge f) \circ(l \wedge l) \sim 0
$$

Thus Corollary 2.2 is proved.

This corollary discribes some homotopy Seifert pairings which may have a Seifert manifold of trivial knot.

2.3. Definition. We shall call two homotopy pairings $\theta_{i}: K_{i} \wedge K_{i} \rightarrow S^{n+1}$, $i=1,2$ contiguous if there exist complexes $L_{1}$ and $L_{2}$, pairings $\alpha: K_{1} \wedge K_{2} \rightarrow S^{n+1}$ and $u: L_{1} \wedge L_{2} \rightarrow S^{n+1}$ from which the latter is a Spanier-Whitehead duality, and an $S$-map $f:\left(K_{1} \vee K_{2}\right) \rightarrow\left(L_{1} \vee L_{2}\right)$ which is a stable homotopy equivalence and such that $\eta \circ(f \wedge f)$ is stably homotopic to $\xi$, where the homotopy pairings

$$
\begin{aligned}
& \eta:\left(L_{1} \vee L_{2}\right) \wedge\left(L_{1} \vee L_{2}\right) \rightarrow S^{n+1} \text { and } \\
& \xi:\left(K_{1} \vee K_{2}\right) \wedge\left(K_{1} \vee K_{2}\right) \rightarrow S^{n+1}
\end{aligned}
$$

are given by the following matrices

$$
\left(\begin{array}{ll}
0 & u \\
0 & 0
\end{array}\right) \text { and }\left(\begin{array}{cc}
\theta_{1} & \alpha \\
(-1)^{n} \alpha^{\prime} & (-1)^{n} \theta_{2}^{\prime}
\end{array}\right) \text {, }
$$

respectively. (The latter means that $\eta \circ\left(l_{i} \wedge l_{j}\right)=a_{i j}$, $\xi \circ\left(k_{i} \wedge k_{j}\right)=b_{i j}$, where $i=1,2, l_{i}: L_{i} \rightarrow L_{1} \vee L_{2}$ and $k_{i}: K_{i} \rightarrow K_{1} \vee K_{2}$ are inclusions, and $a_{i j}$ and $b_{i j}$ are the elements of corresponding matrices.) Here prime denotes "transposition", i.e. the composition with the map which changes the position of factors.

The following two theorems are main results of this section. They state that the contiguity relation of homotopy Seifert pairings corresponds to some simple geometric situation (geometric contiguity) for Seifert manifolds.

2.4. TheOREM. Let $N^{n+2}$ be a compact submanifold of the sphere $S^{n+2}$. Suppose that $\partial N=V_{1}^{n+1} \cup U \cup V_{2}^{n+1}$, where $U=\Sigma^{n} \times[0,1], V_{1} \cap U=\partial V_{1}=\Sigma^{n} \times 0$, $V_{2} \cap U=\partial V_{2}=\Sigma^{n} \times 1, V_{1} \cap V_{2}=\varnothing$ and $\Sigma^{n}$ is a homotopy sphere (Figure 1). Let us orient $V_{1}$ and $V_{2}$ so that they define different orientations of $\partial N$. Then the corresponding homotopy Seifert pairings $\theta_{i}: V_{i} \wedge V_{i} \rightarrow S^{n+1}, i=1,2$, are contiguous. 


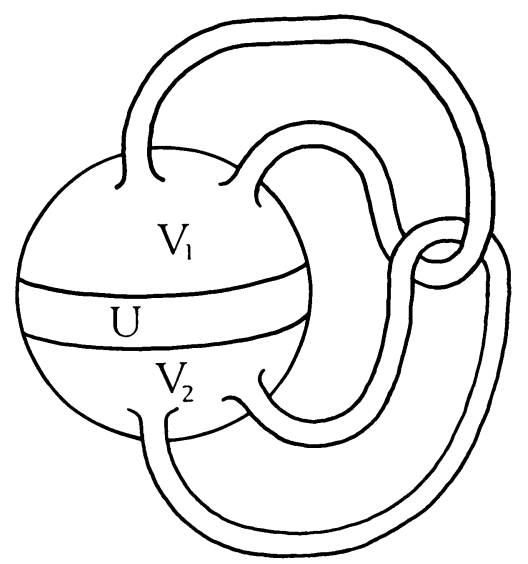

FiguRE 1

Proof. Let us notice that if $\theta$ is a homotopy Seifert pairing of some oriented submanifold $V^{n+1} \subset S^{n+2}$, then $(-1)^{n} \theta^{\prime}$ is the homotopy Seifert pairing of the same submanifold with opposite orientation. It follows from the diagram in the proof of Theorem 1.4.

For the proof of Theorem 2.4 consider the small disk $D^{n+1}$ in int $U=\Sigma^{n} \times$ $(0,1)$. Let $V=\partial N-$ int $D^{n+1}$. We have conditions as in Corollary 2.2 and therefore there is an $S$-map $h: N \vee Y \rightarrow V$ which is stable homotopy equivalent and such that the map $\theta \circ(h \wedge h)$ is given by the matrix $\left(\begin{array}{l}0 \\ 0\end{array}\right)$, where $\theta$ is a homotopy Seifert pairing of manifold $V, u: N \wedge Y \rightarrow S^{n+1}$ is a Spanier-Whitehead duality and $Y=S^{n+2}-$ int $N$.

Let $\omega$ be a simple arc in $U-D^{n+1}$ such that its ends are situated on $\partial V_{1}$ and $\partial V_{2}$. Let $H=V_{1} \cup V_{2} \cup \omega$. The inclusion $g: H \rightarrow V$ is homotopy equivalent. Denote by $i_{1}: V_{1} \rightarrow H$ and $i_{2}: V_{2} \rightarrow H$ the inclusions. Then

$$
\theta_{1}=\theta \circ\left(g \circ i_{1} \wedge g \circ i_{1}\right), \quad(-1)^{n} \theta_{2}^{\prime}=\theta \circ\left(g \circ i_{2} \wedge g \circ i_{2}\right) .
$$

The distinction here is caused by the fact that the orientation of $V_{2}$ is opposite to the orientation of $V$ (see the remark at the beginning of the proof). If by $\alpha$ we denote $\theta \circ\left(g \circ i_{1} \wedge g \circ i_{2}\right)$, then

$$
\theta \circ\left(g \circ i_{2} \wedge g \circ i_{1}\right) \sim(-1)^{n} \alpha^{\prime} .
$$

It follows from these relations that if the space $H$ is identified with $V_{1} \vee V_{2}$ and if by $f$ we denote the composition of the map $g$ and homotopy equivalence $V \rightarrow N \vee Y$ inverse to $h$, then all conditions of Definition 2.3 will be satisfied.

The theorem is proved.

The following theorem states that under some connectivity assumptions the contiguity of homotopy Seifert pairings implies "a geometrical contiguity", i.e. the situation which has been considered in Theorem 2.4.

2.5. TheOREM. Let $V_{1}$ and $V_{2}$ be r-connected $(n+1)$-dimensional compact submanifolds of $S^{n+2}$ and $\theta_{i}: V_{i} \wedge V_{i} \rightarrow S^{n+1}, i=1,2$, are corresponding homotopy Seifert pairings. Suppose that $3 r>n+1>6$. If $\partial V_{i}$ are homotopy spheres and 
pairings $\theta_{1}$ and $\theta_{2}$ are contiguous, then there exist isotopies $g_{t}$ and $h_{t}$ of $S^{n+2}$ and a compact submanifold $N^{n+2} \subset S^{n+2}$ such that

$$
\partial N=g_{1}\left(V_{1}\right) \cup U \cup h_{1}\left(V_{2}\right),
$$

where

$$
\begin{gathered}
U=\sum^{n} \times[0,1], \quad g_{1}\left(V_{1}\right) \cap U=\sum^{n} \times 0=\partial g_{1}\left(V_{1}\right), \\
h_{1}\left(V_{2}\right) \cap U=\partial h_{1}\left(V_{2}\right)=\sum^{n} \times 1, \quad g_{1}\left(V_{1}\right) \cap h_{1}\left(V_{2}\right)=\varnothing
\end{gathered}
$$

and the orientations of $g_{1}\left(V_{1}\right)$ and $h_{1}\left(V_{2}\right)$ define opposite orientations of $\partial N$.

In the proof of this theorem we shall use the following lemma.

2.6. Lemma. Let $2 r+2 \geqslant k>r \geqslant 1$ and.X be a finite complex with $H_{i}(X ; G)=$ 0 for $i \leqslant r$ or $i>k$ and for any abelian group $G$. Then there exists a finite $r$-connected $k$-dimensional complex $Y$ such that $S X$ is homotopy equivalent to $S Y$.

This is the standard result (compare [14, Chapter 8, Exercise D1]).

2.7. Proof of Theorem 2.5. Since the pairings $\theta_{i}: V_{i} \wedge V_{i} \rightarrow S^{n+1}$ are contiguous, then, according to the definition, there exist complexes $L_{1}$ and $L_{2}$, pairings $\alpha$ : $V_{1} \wedge V_{2} \rightarrow S^{n+1}$ and $v: L_{1} \wedge L_{2} \rightarrow S^{n+1}$ (from which the latter is a SpanierWhitehead duality), and an $S$-map $f:\left(V_{1} \vee V_{2}\right) \rightarrow\left(L_{1} \vee L_{2}\right)$ which is stable homotopy equivalent and such that $\eta \circ(f \wedge f)$ is stably homotopic to $\xi$, where the homotopy pairings

$$
\begin{aligned}
& \eta:\left(L_{1} \vee L_{2}\right) \wedge\left(L_{1} \vee L_{2}\right) \rightarrow S^{n+1}, \text { and } \\
& \xi:\left(V_{1} \vee V_{2}\right) \wedge\left(V_{1} \vee V_{2}\right) \rightarrow S^{n+1},
\end{aligned}
$$

are given by matrices

$$
\left(\begin{array}{ll}
0 & v \\
0 & 0
\end{array}\right) \text { and }\left(\begin{array}{cc}
\theta_{1} & \alpha \\
(-1)^{n} \alpha^{\prime} & (-1)^{n} \theta_{2}^{\prime}
\end{array}\right)
$$

respectively.

The constructions of the desirable submanifolds $N$ and isotopies $g_{t}$ and $h_{t}$ are performed in several steps.

Step 1. This is the construction of the $(n+2)$-dimensional compact submanifold $N \subset S^{n+2}$, which satisfies the following condition. If $V^{n+1}$ is obtained from $\partial N$ by removing the interior of some small disk $D^{n+1} \subset \partial N$, then there exists a stable homotopy equivalence $d: L_{1} \vee L_{2} \rightarrow V$ for which $\theta \circ(d \wedge d)$ is homotopic to $\eta$, where $\theta$ is the homotopy Seifert pairing of $V$.

For this, first note that by virtue of Lemma 2.6 and properties of $V_{1}$ and $V_{2}$, we may suppose that $L_{1}$ and $L_{2}$ are $r$-connected complexes of dimension $\leqslant n-r$. Let $\tau: L_{1} \rightarrow N^{n+2}$ be the trivial thickening of $L_{1}$. Let $V=\partial N-$ int $D^{n+1}$ and $Y=$ $S^{n+2}-$ int $N$. Consider stable homotopy equivalence $e: N \vee Y \rightarrow V$ which existence is established in Corollary 2.2. There exists an $S$-map $\sigma: L_{2} \rightarrow Y$ for which $u \circ(\tau \wedge \sigma) \stackrel{S}{\sim} v$, where $u: N \wedge Y \rightarrow S^{n+1}$ is the pairing considered in Corollary 2.2. Since $u$ and $v$ are Spanier-Whitehead dualities, then $\sigma$ is stable homotopy 
equivalent. By virtue of the suspension theorem and our connectivity assumptions we may suppose that $\sigma$ is the usual map and $u \circ(\tau \wedge \sigma) \sim v$. If we take $d$ as the composition

$$
L_{1} \vee L_{2} \stackrel{\tau \vee \sigma}{\rightarrow} N \vee Y \stackrel{e}{\rightarrow} V,
$$

then

$$
\theta \circ(d \wedge d)=\theta \circ(e \wedge e) \circ((\tau \vee \sigma) \wedge(\tau \vee \sigma))=\eta
$$

Step 2. This is the construction of isotopy $l_{t}$ of $S^{n+2}$ such that $V_{1} \cap l_{1}\left(V_{2}\right)=\varnothing$ and the composition of the map $1 \wedge\left(\left.l_{1}\right|_{V_{2}}\right): V_{1} \wedge V_{2} \rightarrow V_{1} \wedge l_{1}\left(V_{2}\right)$ and the canonical pairing $V_{1} \wedge l_{1}\left(V_{2}\right) \rightarrow S^{n+1}$ is homotopic to $\alpha: V_{1} \wedge V_{2} \rightarrow S^{n+1}$.

This may be done in the following way. If $Z$ is the complement of a tubular neighborhood of $V_{1}$ in $S^{n+2}$ and $w: V_{1} \wedge Z \rightarrow S^{n+1}$ is the canonical pairing which is a Spanier-Whitehead duality, then there is a map $\psi: V_{2} \rightarrow Z$ for which $w \circ(1 \wedge$ $\psi) \sim \alpha$. (Here we do not mention the usual arguments about connectivity conditions and application of the suspension theorem.) Now we may construct the thickening $V_{2} \rightarrow N_{2}$ induced by $\psi$. By the same arguments we have seen used in the proof of Theorem 1.3 we can construct the map $V_{2} \rightarrow \partial N_{2}$ such that a thickening $\varphi$ : $V_{2} \rightarrow W_{2}$, induced by this map, has the following property: $\delta \circ(\varphi \wedge \varphi) \sim \theta_{2}$, where $\delta: W_{2} \wedge W_{2} \rightarrow S^{n+1}$ is the homotopy Seifert pairing of $W_{2}$. Also, we shall have that the composition of the map $1 \wedge \varphi: V_{1} \wedge V_{2} \rightarrow V_{1} \wedge W_{2}$ and the canonical pairing $V_{1} \wedge V_{2} \rightarrow S^{n+1}$ is homotopic to $\alpha$. Now Theorem 1.2 is applicable and we obtain the desirable isotopy $l_{t}$ with $l_{1}\left(V_{2}\right)=W_{2}$. See Figure 2 .

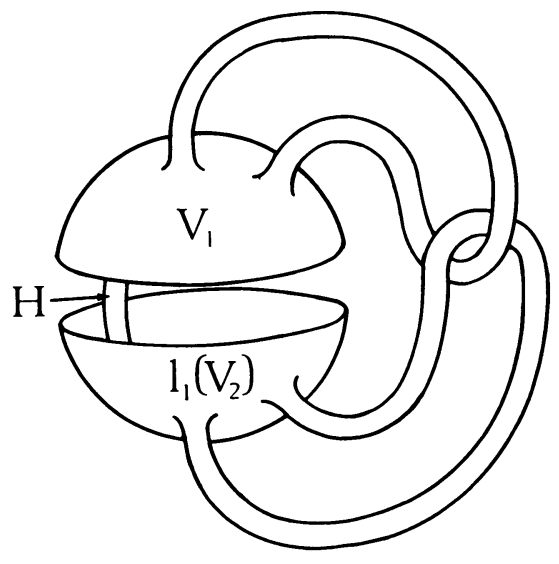

FIGURE 2

Step 3. Let $H$ be an $(n+1)$-dimensional disk in $S^{n+2}$ with the following properties:

(1) $H \cap V_{1}=H \cap \partial V_{1}=\partial H \cap V_{1}=D_{1}$ and $H \cap l_{1}\left(V_{2}\right)=H \cap \partial l_{1}\left(V_{2}\right)=$ $\partial H \cap l_{1}\left(V_{2}\right)=D_{2}$ are two $n$-dimensional disks in $\partial H$.

(2) Orientations of $D_{1}$ and $D_{2}$, defined by that of $\partial V_{1}$ and $\partial l_{1}\left(V_{2}\right)$ respectively, determine the opposite orientations of $\partial H$ (Figure 2). 
Let the manifold $W$ be obtained from $V_{1} \cup H \cup l_{1}\left(V_{2}\right)$ by smoothing corners. Choose the orientation of $W$ according to that of $V_{1}$. Then there is a homotopy equivalence $q: V_{1} \vee V_{2} \rightarrow W$ such that for the homotopy Seifert pairing $\bar{\theta}: W \wedge$ $W \rightarrow S^{n+1}$ of $W$ we shall have that $\bar{\theta} \circ(q \wedge q)$ is given by the matrix

$$
\left(\begin{array}{cc}
\theta_{1} & \alpha \\
(-1)^{n} \alpha^{\prime} & (-1)^{n} \theta_{2}^{\prime}
\end{array}\right),
$$

i.e. the pairing $\bar{\theta} \circ(q \wedge q)$ coincides with $\xi$. Let $F$ be the composition

$$
W \rightarrow V_{1} \vee V_{2} \stackrel{f}{\rightarrow} L_{1} \vee L_{2} \stackrel{d}{\rightarrow} V,
$$

where the first map is homotopy inverse to $q$. (Here we may regard $f$ as usual but not as an $S$-map by virtue of our connectivity assumptions.) Then $F$ is homotopy equivalent and $\theta \circ(F \wedge F) \sim \bar{\theta}$. Applying Theorem 1.2 we get an isotopy $m_{t}$ : $S^{n+2} \rightarrow S^{n+2}$ such that $m_{1}(W)=V$.

Now we may put

$$
g_{t}=m_{t}, \quad h_{t}=m_{t} \circ l_{t} .
$$

Then $g_{1}\left(V_{1}\right)$ and $h_{1}\left(V_{2}\right)$ are disjoint submanifolds of $V$. Let $U=\partial N-$ int $g_{1}\left(V_{1}\right)-$ int $h_{1}\left(V_{2}\right)$. Then it is easy to see that $U$ is the $h$-cobordism between $\partial g_{1}\left(V_{1}\right)$ and $\partial h_{1}\left(V_{2}\right)$ and the $h$-cobordism theorem implies the desired result.

2.8. COROllary. Under the assumption of Theorem 2.5 the oriented knots $\left(S^{n+2}, \partial V_{i}\right), i=1,2$ are equivalent.

The equivalence of these knots is realized by isotopy of sphere $S^{n+2}$ which translates $\Sigma^{n} \times 0$ on $\Sigma^{n} \times 1$ along $U$.

The next statement will establish the reflexivity of the contiguity relation.

2.9. Proposition. If $K$ is a finite polyhedron and $\theta: K \wedge K \rightarrow S^{n+1}$ is the homotopy pairing for which $\theta+(-1)^{n+1} \theta^{\prime}$ is a Spanier-Whitehead duality, then $\theta$ is contiguous to itself.

Proof. By virtue of Spanier-Whitehead theory there are $S$-maps $f, g: K \rightarrow K$ for which

$$
\left(\theta+(-1)^{n+1} \theta^{\prime}\right) \circ(f \wedge 1) \stackrel{S}{\sim} \theta, \quad\left(\theta+(-1)^{n+1} \theta^{\prime}\right) \circ(g \wedge 1) \stackrel{S}{\sim}(-1)^{n} \theta^{\prime} .
$$

Since $\left(\theta+(-1)^{n+1} \theta^{\prime}\right) \circ((f-g) \wedge 1) \stackrel{S}{\sim}\left(\theta+(-1)^{n+1} \theta^{\prime}\right)$, then $f-g \stackrel{S}{\sim} 1_{K}$. Here $1_{K}$ is the identity mapping of $K$. Let $f \vee g: K \vee K \rightarrow K$ be the $S$-map determined by $f$ and $g$; let $e: K \vee K \rightarrow K$ be a map which identifies the summands of the bouquet; and let $i_{1}, i_{2}: K \rightarrow K \vee K$ be a natural inclusion. Determine an $S$-map $h: K \vee K$ $\rightarrow K \vee K$ by the formula $h=i_{1} \circ(f \vee g)+i_{2} \circ e$. Let us prove that $h$ is a stable homotopy equivalence. For this it is enough that $h$ induces the surjective map of integral homology. If $z_{1}, z_{2} \in H_{r} K$, then

$$
h_{*}\left(i_{1 *} z_{1}+i_{2 *} z_{2}\right)=i_{1 *}\left(f_{*} z_{1}+g_{*} z_{2}\right)+i_{2 *}\left(z_{1}+z_{2}\right) \text {. }
$$

Any class $a \in H_{r}(K \vee K)$ may be uniquely presented in the form $a=i_{1 *} a_{1}+$ $i_{2 *} a_{2}$, where $a_{1}, a_{2} \in H_{r} K$. Denote $z_{1}=a_{1}-g_{*} a_{2}, z_{2}=f_{*} a_{2}-a_{1}$. Then it is not 
difficult to verify that $h_{*}\left(i_{1 *} z_{1}+i_{2 *} z_{2}\right)=i_{1 *} a_{1}+i_{2 *} a_{2}=a$. Therefore $h_{*}$ is an epimorphism and $h$ is a stable homotopy equivalence.

Let $\eta:(K \vee K) \wedge(K \vee K) \rightarrow S^{n+1}$ be a homotopy pairing given by the following matrix:

$$
\left(\begin{array}{cc}
0 & \theta+(-1)^{n+1} \theta^{\prime} \\
0 & 0
\end{array}\right)
$$

We have

$$
\begin{aligned}
\eta \circ(h \wedge h)= & \eta \circ\left(\left(i_{1} \circ(f \vee g)+i_{2} \circ e\right) \wedge\left(i_{1} \circ(f \vee g)+i_{2} \circ e\right)\right) \\
= & \eta \circ\left(i_{1} \circ(f \vee g) \wedge i_{1} \circ(f \vee g)\right)+\eta \circ\left(i_{1} \circ(f \vee g) \wedge i_{2} \circ e\right) \\
& +\eta \circ\left(i_{2} \circ e \wedge i_{1} \circ(f \vee g)\right)+\eta \circ\left(i_{2} \circ e \wedge i_{2} \circ e\right) \\
= & \eta \circ\left(i_{1} \circ(f \vee g) \wedge i_{2} \circ e\right)=\left(\theta+(-1)^{n+1} \theta^{\prime}\right) \circ((f \vee g) \wedge e) .
\end{aligned}
$$

From this it follows that

$$
\begin{aligned}
\eta \circ(h \wedge h) \circ\left(i_{1} \wedge i_{1}\right) & =\left(\theta+(-1)^{n+1} \theta^{\prime}\right) \circ((f \vee g) \wedge e) \circ\left(i_{1} \wedge i_{2}\right) \\
& =\left(\theta+(-1)^{n+1} \theta^{\prime}\right) \circ(f \wedge 1) \stackrel{s}{\sim} \theta
\end{aligned}
$$

Analogously one can get

$$
\begin{aligned}
& \eta \circ(h \wedge h) \circ\left(i_{1} \wedge i_{2}\right) \stackrel{s}{\sim} \theta, \\
& \eta \circ(h \wedge h) \circ\left(i_{2} \wedge i_{1}\right) \stackrel{s}{\sim}(-1)^{n} \theta^{\prime}, \\
& \eta \circ(h \wedge h) \circ\left(i_{2} \wedge i_{2}\right) \stackrel{s}{\sim}(-1)^{n} \theta^{\prime},
\end{aligned}
$$

i.e. the pairing $\eta \circ(h \wedge h)$ is given by the following matrix:

$$
\left(\begin{array}{cc}
\theta & \theta \\
(-1)^{n} \theta^{\prime} & (-1)^{n} \theta^{\prime}
\end{array}\right)
$$

This proves the proposition (in the notations of Definition 2.3, $L_{1}=L_{2}=K$, $\left.u=\theta+(-1)^{n+1} \theta^{\prime}, \alpha=\theta\right)$.

\section{The classification of knots.}

3.1. Let $K_{r, n}$ be the set of isotopy types of $n$-dimensional knots $\left(S^{n+2}, k^{n}\right)$ such that $\pi_{i}\left(S^{n+2}-k^{n}\right)=\pi_{i}\left(S^{1}\right)$ for $i<r$. There is the natural map $M_{r, n} \rightarrow K_{r, n}$ which assigns to a submanifold from $M_{r, n}$ its boundary (for the definition of the set $M_{r, n}$ see 1.11). J. Levine proved that this map is surjective. In Corollary 1.12 the map $M_{r, n} \rightarrow Z_{r, n}$ was constructed and it was shown that it is bijective if $3 r>n+1>6$. Thus, if $3 r \geqslant n+1 \geqslant 6$, then we have the map $\Phi: Z_{r, n} \rightarrow K_{r, n}$ which is the composition of the map inverse to the bijection of Corollary 1.12 and the map $M_{r, n} \rightarrow K_{r, n}$ defined above.

The map $\Phi$ is surjective. For the description of the set $K_{r, n}$ we shall find the equivalence relation which arises on $Z_{r, n}$ by virtue of mapping $\Phi$.

3.2. Definition. We shall call two pairs $(K, \theta)$ and $(L, \eta)$, representing two elements of $Z_{r, n}, R$-equivalent if there exists a finite sequence of pairs $\left(K_{1}, \theta_{1}\right), \ldots,\left(K_{s}, \theta_{s}\right)$, where for $1<m<s, K_{m}$ is an $r$-connected complex and $\theta_{m}$ : $K_{m} \wedge K_{m} \rightarrow S^{n+1}$ is a pairing for which $\theta_{m}+(-1)^{n+1} \theta_{m}^{\prime}$ is a Spanier-Whitehead 
duality, such that $\left(K_{1}, \theta_{1}\right)=(K, \theta),\left(K_{s}, \theta_{s}\right)=(L, \eta)$, and pairings $\theta_{m}$ and $\theta_{m+1}$ are contiguous for all $1 \leqslant m \leqslant s-1$.

In other words $R$-equivalence is the equivalence relation on $Z_{r, n}$ which is generated by the contiguity relations. Note that contiguity is reflexive and a symmetric relation; it is not transitive.

3.3. TheOREM. Let $V_{1}$ and $V_{2}$ be oriented $r$-connected $(n+1)$-dimensional compact submanifolds of sphere $S^{n+2}$ and $\theta_{i}: V_{i} \wedge V_{i} \rightarrow S^{n+1}, i=1,2$, are the corresponding Seifert pairings. If $\partial V_{i}$ are homotopy spheres, $3 r \geqslant n+1 \geqslant 6$ and pairs $\left(V_{i}, \theta_{i}\right)$ are $R$-equivalent in $Z_{r, n}$, then the oriented knots $\left(S^{n+2}, \partial V_{i}\right), i=1,2$, are equivalent.

Proof. Since the pairs $\left(V_{1}, \theta_{1}\right)$ and $\left(V_{2}, \theta_{2}\right)$ are $R$-equivalent in $Z_{r, n}$, then according to the definition the finite sequence of pairs $\left(W_{1}, \eta_{1}\right)=\left(V_{1}, \theta_{1}\right)$, $\left(W_{2}, \eta_{2}\right), \ldots,\left(W_{s}, \eta_{s}\right)=\left(V_{2}, \theta_{2}\right)$ exists, where $W_{m}$ is a finite $r$-connected cell complex and $\eta_{m}: W_{m} \wedge W_{m} \rightarrow S^{n+1}$ is the homotopy pairing, $m=\overline{1, s}$, and all conditions formulated in Definition 3.2 are fulfilled. By virtue of Theorem 1.3 and Theorem 1.4 we can assume that $W_{m}$ is a compact oriented $(n+1)$-dimensional submanifold of sphere $S^{n+2}$ for which $\partial W_{m}$ is a homotopy sphere and $\eta_{m}$ is a homotopy Seifert pairing of $W_{m}, m=\overline{1, s}$. Since $\eta_{m}$ and $\eta_{m+1}$ are contiguous and $3 r \geqslant n+1 \geqslant 6$, then by virtue of Corollary 2.8 , the oriented knots $\left(S^{n+2}, \partial W_{m}\right)$ and $\left(S^{n+2}, \partial W_{m+1}\right)$ are equivalent, $m=\overline{1, s-1}$. Therefore the knot $\left(S^{n+2}, \partial W_{1}\right)$ $=\left(S^{n+2}, \partial V_{1}\right)$ is equivalent to the $\operatorname{knot}\left(S^{n+2}, \partial W_{s}\right)=\left(S^{n+2}, \partial V_{2}\right)$.

The theorem is proved.

3.4. THEOREM. Let $V_{1}$ and $V_{2}$ be oriented $r$-connected $(n+1)$-dimensional compact submanifolds of sphere $S^{n+2}$ and $\theta_{i}: V_{i} \wedge V_{i} \rightarrow S^{n+1}, i=1,2$, are the corresponding homotopy Seifert pairings where $r \geqslant 2, n \geqslant 4$. If $\partial V_{i}$ are homotopy spheres and the oriented knots $\left(S^{n+2}, \partial V_{i}\right), i=1,2$, are equivalent, then the pairs $\left(V_{i}, \theta_{i}\right), i=1,2$, are $R$-equivalent in $Z_{r, n}$.

REMARK. The theorem is true without the assumptions that $r \geqslant 2, n \geq 4$. This more general statement may be deduced from the given theorem with the help of G. Bredon's suspension construction which permits us "to increase" $r$ and $n$. In the present paper this more general statement will not be used.

The proof of Theorem 3.4 will be based on the following lemmas.

We shall suppose below that $n \geqslant 2 r+1$ otherwise $V_{1}$ and $V_{2}$ must be contractible and the theorem becomes trivial.

3.5. LEMMA. Under the assumptions of Theorem 3.4 there is an r-connected $(n+2)$-dimensional compact submanifold $V$ (with corners) of $S^{n+2} \times[1,2]$ such that

$$
\begin{aligned}
V \cap S^{n+2} \times \nu & =V_{\nu} \times \nu, \quad \nu=1,2, \quad \text { and } \\
\partial V & =V_{1} \times 1 \cup V_{2} \times 2 \cup X,
\end{aligned}
$$

where $X$ is the trace of isotopy translating $\partial V_{1}$ on $\partial V_{2}$.

Proof. The existence of $V$ satisfying all required conditions besides $r$-connectness was proved by $J$. Levine [10, p. 186]. In order to construct an $r$-connected $V$ one may use the method of modification described in $\$ \S 4$ and 5 of [7]. This method 
does not change $\partial V$ and gives an $r$-connected manifold by virtue of analogy of Lemma 4 of [7] and the fact that $\pi_{i}\left(S^{n+2} \times[1,2]-X\right)=\pi_{i}\left(S^{1}\right)$ for $i<r$.

Later on we shall identify $V_{\nu}$ with $V_{\nu} \times \nu \subset S^{n+2} \times[1,2]$, where $\nu=1,2$.

3.6. Lemma. Let $V$ be the same as in Lemma 3.5 and let $Y$ be the closure of the complement of the tubular neighborhood of $V$ in $S^{n+2} \times[1,2]$. Denote $Y_{v}=Y \cap$ $S^{n+2} \times \nu, \nu=1,2$. Fix some orientation of $V$ and let $i_{+}, i_{-}:\left(V ; V_{1}, V_{2}\right) \rightarrow$ $\left(Y ; Y_{1}, Y_{2}\right)$ be translations along the fields of positive and negative normals to $V$. If for some $k$ the homomorphisms

$$
i_{+*}, i_{-*}: T_{k}\left(V, V_{1}\right) \rightarrow T_{k}\left(Y, Y_{1}\right)
$$

are both monomorphisms, then $T_{k}\left(V, V_{1}\right)=0=T_{k}\left(Y, Y_{1}\right)$, where $T_{k}$ denotes the torsion subgroup of $k$-dimensional homology. Besides, if $T_{k}\left(V, V_{1}\right)=0$ and $H_{k}\left(V, V_{1}\right)$ $\neq 0$, then there exists an indivisible element $\alpha \in H_{k}\left(V, V_{1}\right), \alpha \neq 0$ for which $i_{+*}(\alpha)$ $=0$ or $i_{-*}(\alpha)=.0$.

Proof. The infinite cyclic covering $p: \tilde{Z} \rightarrow S^{n+2} \times[1,2]-X$ may be constructed from the infinite sequence of copies of the space $Y$ by the same way as the infinite cyclic covering of a knot may be constructed by cutting a sphere along a Seifert manifold [9]. Let $\tilde{Z}_{1}=p^{-1}\left(\left(S^{n+2} \times 1\right)-\partial V_{1}\right)$. Since $X$ is the trace of isotopy, it follows that $H_{*}\left(Z, Z_{1}\right)=0$ and the arguments analogous to that used in $[9$, p. 541] prove that there is an isomorphism of $\Lambda$-modules

$$
d: H_{k}\left(V, V_{1}\right) \otimes \Lambda \rightarrow H_{k}\left(Y, Y_{1}\right) \otimes \Lambda,
$$

where

$$
d(a \otimes 1)=i_{+*}(a) \otimes t-i_{-*}(a) \otimes 1, \quad a \in H_{k}\left(V, V_{1}\right) .
$$

Here $\Lambda=Z\left[t, t^{-1}\right]$ is the group ring of the infinite cyclic group.

Suppose that $i_{+*}, i_{-*}: T_{k}\left(V, V_{1}\right) \rightarrow T_{k}\left(Y, Y_{1}\right)$ are both monomorphisms. Let $b \in T_{k}\left(Y, Y_{1}\right), b \neq 0$. Then there is a unique element $q \in H_{k}\left(V, V_{1}\right) \otimes \Lambda$ for which $d(q)=b \otimes 1$. Write $q$ in the form

$$
q=\sum_{i=m}^{n} z_{i} \otimes t^{i},
$$

where $m \leqslant n, z_{i} \in H_{k}\left(V, V_{1}\right)$. We may suppose that $z_{n} \neq 0$. Since $d$ is an isomorphism, then $z_{i}$ must belong to $T_{k}\left(V, V_{1}\right)$.

The two following cases are possible: (1) $n>0$, (2) $n<0$. If $n>0$, then $d(q)$ contains as summand the element $i_{+*}\left(z_{n}\right) \otimes t^{n+1}$ and does not contain other elements of degree $n+1$. Since $\Lambda$ is a free abelian group, then it follows from equality $d(q)=b \otimes 1$ that $i_{+*}\left(z_{n}\right)=0$. Since $i_{+*}$ is a monomorphism, then $z_{n}=0$ and we have obtained a contradiction. Analogously case (2) may be reduced to a contradiction by using that $i_{-*}$ is a monomorphism. So $T_{k}\left(Y, Y_{1}\right)=0$ and then $T_{k}\left(V, V_{1}\right)=0$.

This proves the first statement of the lemma.

In order to prove the second statement let us notice that if $H_{k}\left(Y, Y_{1}\right) \neq 0$, then at least one of the homomorphisms

$$
i_{+*}, i_{-*}: H_{k}\left(V, V_{1}\right) \rightarrow H_{k}\left(Y, Y_{1}\right)
$$


must have nontrivial kernel. This may be deduced similarly to recently described arguments from the fact that $d$ is an isomorphism. If $T_{k}\left(V, V_{1}\right)=0$, then, as it has been proved above, the group $H_{k}\left(Y, Y_{1}\right)$ has no torsion. From this follows that if $a \in \operatorname{ker} i_{+*}$ and $a=p c$, where $p \in Z, p \neq 0$ and $c$ is indivisible, then $c \in \operatorname{ker} i_{+*}$. This implies the second statement of the lemma.

It is clear that in this lemma we may replace $V_{1}$ by $V_{2}$ and $Y_{1}$ by $Y_{2}$.

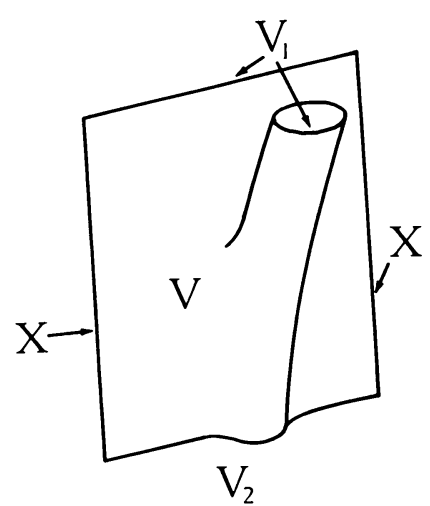

a.

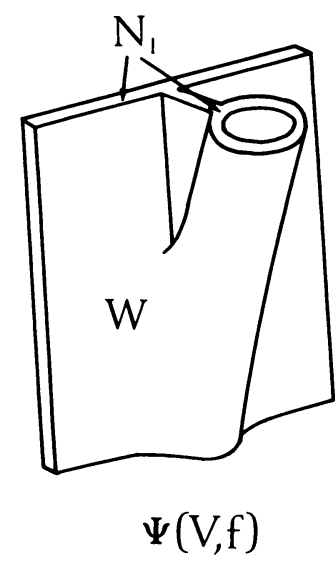

b.

Figure 3

3.7. Let $V^{n+2}$ be a manifold, the boundary of which is the union of three $(n+1)$-manifolds $V_{1}, V_{2}, X$ and has the corners along $\partial V_{1}$ and $\partial V_{2}$ (Figure 3a). Let $f:\left(D_{+}^{k}, \partial D_{+}^{k}\right) \times D^{n+2-k} \rightarrow\left(V, V_{1}\right)$ be a smooth imbedding, where $D_{+}^{k}$ is the upper semisphere of $S^{k}$. Consider the manifold $\psi(V, f)$ which is obtained from the union $V \times[0,1] \cup D^{k+1} \times D^{n+2-k}$ by identification of points $(x, y) \in D_{+}^{k} \times$ $D^{n+2-k}$ with $(f(x, y), 1)$ (see Figure $3 b$ ) and then smoothing corners along $X \times 1$, $\left(f\left(D_{+}^{k} \times S^{n+1-k}\right), 1\right)$ and $\left(f\left(\partial D_{+}^{k} \times D^{n+2-k}\right), 1\right)$. Let

$$
N_{1}=V_{1} \times[0,1] \cup D_{-}^{k} \times D^{n+2-k},
$$

where $D_{-}^{k}$ is the low semisphere of $S^{k}$ and the points $(x, y) \in \partial D_{-}^{k} \times D^{n+2-k}$ are identified with $(f(x, y), 1)$. Let

$$
N_{2}=V_{2} \times[0,1]
$$

Then

$$
\partial \psi(V, f)=V \cup N_{1} \cup N_{2} \cup W,
$$

where $V$ is identified with $V \times \theta$, and $W$ is an $(n+2)$-dimensional manifold with $\partial W=W_{1} \cup W_{2} \cup X$, where $W_{\nu}=W \cap N_{\nu}, \nu=1,2$.

Thus we have obtained $\left(\psi(V, f) ; V, N_{1}, N_{2}, W\right)$.

Of course, the manifold $\psi(V, f)$ is homeomorphic to $V \times[0,1]$, but for us the given definition is more convenient. 
3.8. LEMMA. Let $V$ be the $(n+2)$-dimensional $r$-connected submanifold of $S^{n+2} \times$ $[1,2]$, constructed in Lemma 3.5. Let $\alpha \in \pi_{k}\left(V, V_{1}\right)$ be such an element that $i_{+*} \alpha=0 \in \pi_{k}\left(Y, Y_{1}\right)$, where $Y, Y_{1}, i_{+}$are the same as in Lemma 3.6. Suppose that $k \leqslant n-1,2 k \leqslant n+r$. Then there exist an imbedding

$$
f:\left(D_{+}^{k}, \partial D_{+}^{k}\right) \times D^{n+2-k} \rightarrow\left(V, V_{1}\right),
$$

which realizes $\alpha$, and an imbedding

$$
g: \psi(V, f) \rightarrow S^{n+2} \times[1,2],
$$

extending the imbedding of $V$ and such that

$$
g(\psi(V, f)) \cap S^{n+2} \times \nu=N_{\nu}, \quad \nu=1,2 .
$$

Proof. The possibility to realize $\alpha$ by imbedding $\varphi:\left(D_{+}^{k}, \partial D_{+}^{k}\right) \rightarrow\left(V, V_{1}\right)$ follows from Corollary 1.1 of Hudson's work [3]. Since $i_{+*}(\alpha)=0$, then there exists a continuous map $h: D^{k+1} \rightarrow S^{n+2} \times[1,2]$ extending $\varphi$ and such that

$$
\begin{aligned}
h\left(D^{k+1}\right) \cap S^{n+2} \times 1 & =h\left(D_{-}^{k}\right), \\
h\left(D^{k+1}\right) \cap S^{n+2} \times 2 & =\varnothing, \\
h\left(D^{k+1}\right) \cap V & =h\left(D_{+}^{k}\right) .
\end{aligned}
$$

By virtue of Theorem 1 of [3], applied to the manifold, which has been obtained from $S^{n+2} \times[1,2]$ by cutting along $V$ and smoothing corners, we may suppose that $h$ is an imbedding. Now we may obtain the desired imbedding $g$ by thickening $V$ and $h\left(D^{k+1}\right)$ in $S^{n+2} \times[1,2]$.

This proves the lemma.

It is obvious that the analogy construction and statement are true if $i_{+}$is replaced by $i_{-}$or $V_{1}$ by $V_{2}$.

This lemma will enable us to prove Theorem 3.4 considering submanifold $g(W) \subset S^{n+2} \times[1,2]$ instead of $V$, since $\partial V_{1}=\partial g\left(W_{1}\right)$ and $V_{1}$ and $g\left(W_{1}\right)$ have contiguous homotopy Seifert pairings (as it follows from Theorem 2.4). The same is true for homotopy Seifert pairings of $V_{2}$ and $g\left(W_{2}\right)$. We shall say that $g(W)$ is obtained by killing $\alpha$.

3.9. Lemma. Let $V, f$ and $W$ be the same as in 3.7 and let $\alpha \in \pi_{k}\left(V, V_{1}\right)$ be the homotopy class realized by imbedding $f$. Let $\beta \in \pi_{n+1-k}\left(W, W_{2}\right)$ be the homotopy class realized by sphere $x_{0} \times S^{n+1-k} \subset W$, where $x_{0} \in D^{k+1}$. Then

(a) $H_{i}\left(W, W_{1}\right) \approx H_{i}\left(V, V_{1}\right)$ for $i<k$;

(b) $H_{k}\left(W, W_{1}\right) \approx H_{k}\left(V, V_{1}\right) /(h(\alpha))$, where $h$ denotes the Hurewicz homomorphism and $(h(\alpha))$ is the subgroup generated by $h(\alpha)$;

(c) $H_{j}\left(W, W_{2}\right) \approx H_{j}\left(V, V_{2}\right)$ for $j<n+1-k$;

(d) $H_{n+1-k}\left(V, V_{2}\right) \approx H_{n+1-k}\left(W, W_{2}\right) /(h(\beta))$;

(e) if $h(\alpha)$ has a finite order, then $h(\beta)$ is an element of infinite order;

(f) if $h(\alpha)$ is an indivisible element, then $h(\beta)=0$;

(g) if $V, V_{1}, V_{2}$ are $r$-connected and $r<k<n+1-r$, then $W, W_{1}, W_{2}$ are also $r$-connected. 
Proof. It is easy to see that the pair $\left(W, W_{1}\right)$ is homeomorphic to $\left(V-f\left(D_{+}^{k} \times\right.\right.$ int $\left.D^{n+2-k}\right),\left(V_{1}-f\left(\partial D_{+}^{k} \times\right.\right.$ int $\left.\left.\left.D^{n+2-k}\right) \cup f\left(D_{+}^{k} \times \partial D^{n+2-k}\right)\right)\right)$. The inclusion of the last pair in $\left(V, V_{1} \cup f\left(D_{+}^{k} \times D^{n+2-k}\right)\right)$ is an excision map and induces the isomorphism of homology groups. Thus

$$
H_{*}\left(W, W_{1}\right) \approx H_{*}\left(V, V_{1} \cup f\left(D_{+}^{k} \times D^{n+2-k}\right)\right) .
$$

Now (a) and (b) follow from consideration of the corresponding homology sequence.

Let us note that $\left(W, W_{2}\right)$ is homeomorphic to $\left(V-f\left(D_{+}^{k} \times\right.\right.$ int $\left.\left.D^{n+2-k}\right), V_{2}\right)$ and now the inclusion of the last pair to $\left(V, V_{2}\right)$ gives the homomorphisms $H_{j}\left(W, W_{2}\right) \rightarrow H_{j}\left(V, V_{2}\right)$. From this one may easily deduce (c) and (d).

Let us prove (e). We shall identify $\left(W, W_{2}\right)$ with $\left(V-f\left(D_{+}^{k} \times\right.\right.$ int $\left.\left.D^{n+2-k}\right), V_{2}\right)$ by means of the evident homeomorphism. Then $h(\beta)$ will be identified with the homology class which is realized by $f\left(x_{0} \times S^{n+1-k}\right)$, where $x_{0} \in D_{+}^{k}$. Suppose that $h(\beta)$ has finite order $p$, say. Then there is an $(n+2-k)$-chain $c$ in $V-$ $f\left(D_{+}^{k} \times\right.$ int $\left.D^{n+2-k}\right)$ such that

$$
\partial c=p z_{0}+u
$$

where $z_{0}$ is the fundamental circle of $f\left(x_{0} \times S^{n+1-k}\right)$ and $u$ is some chain in $V_{2}$. Denote by $y_{0}$ the chain in $f\left(x_{0} \times D^{n+2-k}\right)$ for which $\partial y_{0}=z_{0}$. Let $c_{1}=p y_{0}-c$. Then $c_{1}$ is a circle module $V_{2}$ and the intersection number of $h(\alpha)$, and $\left\{c_{1}\right\}$ is equal to $\pm p \neq 0$. But it is impossible if $h(\alpha)$ has a finite order. This proves (e).

If $h(\alpha)$ is an indivisible element, then Poincare duality implies that there is a homology class $u \in H_{n+2-k}(V)$ such that the intersection number $h(\alpha) \cdot u$ is equal to 1 . We may realize $u$ by a circle of the form $a+b$, where $a$ is a chain in $f\left(D_{+}^{k} \times D^{n+2-k}\right)$ and $b$ is a chain in $V-f\left(D_{+}^{k} \times\right.$ int $\left.D^{n+2-k}\right)$. It is clear that $a$ determines the homology class of the pair $\left(f\left(D_{+}^{k} \times D^{n+2-k}\right), f\left(D_{+}^{k} \times S^{n+1-k}\right)\right)$ which is homologous to $y_{0}$. Thus there is some $(n+2-k)$-chain $e$ in $f\left(D_{+}^{k} \times S^{n+1-k}\right)$ such that $\partial e=z_{0}-\partial a$. Consider $e+b$. It is a chain in $V-$ $f\left(D_{+}^{k} \times\right.$ int $\left.D^{n+2-k}\right)$ and its boundary is equal to $z_{0}$. Therefore $z_{0}$ is homologous to zero and so $h(\beta)=0$. This proves (f).

Assertion (g) is standard and well known.

3.10. Lemma. Suppose we have conditions as in Theorem 3.4. Let $q$ be some integer, $2 q \leqslant n$. Then there is an $(n+2)$-dimensional compact submanifold $W$ (with corners) of $S^{n+2} \times[1,2]$, satisfying the following conditions:

(a) $\partial W=W_{1} \cup W_{2} \cup X$, where $W_{\nu}=W \cap S^{n+2} \times \nu, \nu=1,2$, and $X$ is the trace of isotopy, translating $\partial V_{1}$ and $\partial V_{2}$;

(b) manifolds $W, W_{1}, W_{2}$ are r-connected;

(c) from (a). It follows that $\partial W_{\nu}=\partial V_{\nu}, \nu=1,2$, and if we orient $W_{\nu}$ so that this equality will be true as for oriented manifolds, then homotopy Seifert pairings of $W_{\nu}$ and $V_{\nu}$ are $R$-equivalent in $Z_{r, n}, \nu=1,2$;

(d) $H_{i}\left(W, W_{\nu}\right)=0$ for $i \leqslant q, \nu=1,2$. 
Proof. We construct $W$ by induction. First we have the manifold $V$ constructed in Lemma 3.5. This manifold satisfies all required conditions for $q=r$.

Consider the group $\pi_{r+1}\left(V, V_{1}\right)=H_{r+1}\left(V, V_{1}\right)$. If this group is nonzero, then by virtue of Lemma 3.6 there is $\alpha \in H_{r+1}\left(V, V_{1}\right), \alpha \neq 0$ for which $i_{+*} \alpha=0$ or $i_{-*} \alpha=0$. Lemma 3.8 enables us to construct manifold $V^{\prime}$ obtained from $V$ by killing $\alpha$. Then $V^{\prime}$ will satisfy the condition (a) (as it follows from the construction) and (b) (by virtue of 3.9(g)). The condition (c) will also be satisfied (it follows from Theorem 2.4, see remark after proof of Lemma 3.8). Besides

$$
\begin{array}{lll}
H_{i}\left(V^{\prime}, V_{1}^{\prime}\right)=0 & \text { for } i \leqslant r & (\text { see 3.9(a)); } \\
H_{j}\left(V^{\prime}, V_{2}^{\prime}\right)=0 & \text { for } j \leqslant r & \text { (see 3.9(c)); }
\end{array}
$$

and the group $H_{r+1}\left(V^{\prime}, V_{1}^{\prime}\right)$ will be a proper factor-group of $H_{r+1}\left(V, V_{1}\right)$ (by virtue of 3.9(b)).

Since $H_{r+1}\left(V, V_{1}\right)$ is a finitely generated abelian group, this procedure may be iterated a finite number of times after which $i_{+*}$ and $i_{-*}$ will both be monomorphisms. Then, by Lemma 3.6, we shall have $V^{\prime \prime}$ with $H_{i}\left(V^{\prime \prime}, V_{1}^{\prime \prime}\right)=0$ for $i<r+1$.

By the same way we may kill group $H_{r+1}\left(V^{\prime \prime}, V_{2}^{\prime \prime}\right)$ without changing groups $H_{i}\left(V^{\prime \prime}, V_{1}^{\prime \prime}\right)$ for $i \leqslant r+1$ because $2(r+1) \leqslant 2 q \leqslant n$ (see Lemma 3.9(c)).

This shows that we can proceed by induction on $q$.

This completes the proof.

3.11. The previous lemma permits us to kill homology groups below the middle dimension. In order to kill middle dimensional homology groups we must consider two cases.

Case 1. $n$ is even. Let $n=2 q$. Then Lemma 3.10 gives a manifold $W$ and we want to kill groups $H_{q+1}\left(W, W_{1}\right)=\pi_{q+1}\left(W, W_{1}\right)$ and $H_{q+1}\left(W, W_{2}\right)=$ $\pi_{q+1}\left(W, W_{2}\right)$. Since $\partial W=W_{1} \cup W_{2} \cup X$, where $X=\Sigma^{n} \times[1,2]$, Poincaré duality and the universal coefficients theorem imply that these groups are isomorphic and free abelian. If these groups are nonzero, then by virtue of Lemma 3.6 there is an indivisible element $\alpha \in \pi_{q+1}\left(W, W_{1}\right)$ with $i_{+*} \alpha=0$ or $i_{-*} \alpha=0$. If $W^{\prime}$ is obtained by killing $\alpha$, then $h(\beta)=0$ (see Lemma 3.9(f)) and so $H_{q}\left(W^{\prime}, W_{2}^{\prime}\right)=$ $H_{q}\left(W, W_{2}\right)=0$ by Lemma $3.9(\mathrm{~d})$. Besides rank of $H_{q+1}\left(W^{\prime}, W_{1}^{\prime}\right)$ is smaller than rank of $H_{q+1}\left(W, W_{1}\right)$ and so, by a finite number of steps we shall have $W^{\prime \prime}$ with $H_{i}\left(W^{\prime \prime}, W_{1}^{\prime \prime}\right)=0$ for $i \leqslant q+1$ and $H_{j}\left(W^{\prime \prime}, W_{2}^{\prime \prime}\right)=0$ for $j \leqslant q$. But then Poincaré duality implies that

$$
H_{*}\left(W^{\prime \prime}, W_{\nu}^{\prime \prime}\right)=0, \quad \nu=1,2 .
$$

Case 2. $n$ is odd, $n=2 q+1$. Let $W$ be the manifold constructed in Lemma 3.10. If group Tors $\pi_{q+1}\left(W, W_{1}\right)$ is nonzero, then by Lemma 3.6 this group contains $\alpha \neq 0$ with $i_{+*} \alpha=0$ or $i_{-*} \alpha=0$. Let $W^{\prime}$ be obtained from $W$ by killing $\alpha$. Then $W^{\prime}$ also satisfies the conditions of Lemma 3.10 and Tors $H_{q+1}\left(W^{\prime}, W_{1}^{\prime}\right)$ is a proper factor-group of Tors $H_{q+1}\left(W, W_{1}\right)$. Thus after a finite number of such operations we obtain manifold $W^{\prime \prime}$ with Tors $H_{q+1}\left(W^{\prime \prime}, W_{1}^{\prime \prime}\right)=0$. Now we may kill by the same way the group Tors $H_{q+1}\left(W^{\prime \prime}, W_{2}^{\prime \prime}\right)$. Under this, the group $H_{q+1}\left(W^{\prime \prime}, W_{1}^{\prime \prime}\right)$ can increase, but its torsion subgroup will remain zero as it follows from Lemma 3.9(e). So we shall obtain submanifold $W^{\prime \prime \prime}$ satisfying all conditions of Lemma 3.10 
and

$$
\text { Tors } H_{q+1}\left(W^{\prime \prime \prime}, W_{1}^{\prime \prime \prime}\right)=\text { Tors } H_{q+1}\left(W^{\prime \prime \prime}, W_{2}^{\prime \prime \prime}\right)=0 \text {. }
$$

After this, indivisible elements of groups $H_{q+1}\left(W^{\prime \prime \prime}, W_{1}^{\prime \prime}\right)$ and $H_{q+1}\left(W^{\prime \prime \prime}, W_{2}^{\prime \prime \prime}\right)$ may be killed as in Case 1 (by virtue of Lemmas 3.6 and 3.9(f)). Now Poincaré duality implies that we have killed all relative homology groups.

Thus in both cases we have proved the following:

3.12. LEMMA. Under assumptions of Theorem 3.4 there is an $(n+2)$-dimensional compact submanifold $W$ (with corners) of $S^{n+2} \times[1,2]$ which has properties (a), (b), (c) of Lemma 3.10 and

$$
H_{*}\left(W, W_{\nu}\right)=0 \text { for } \nu=1,2 \text {. }
$$

3.13. Proof of Theorem 3.4. Let $W$ be a submanifold constructed as in Lemma 3.12. Then $W$ is a relative $h$-cobordism and, by virtue of the $h$-cobordism theorem, the pair $(W, X)$ is diffeomorphic to $\left(W_{1}, \partial W_{1}\right) \times[1,2]$. So we obtain a concordance between $W_{1}$ and $W_{2}$. By theorems of Rourke [11], there is an isotopy of $S^{n+2} \times[1,2]$ carrying $W$ on $W_{1} \times[1,2]$. Thus we obtain an isotopy of $S^{n+2}$ carrying $W_{2}$ on $W_{1}$ with preservation of orientations, specified in Lemma 3.10(c). So the homotopy Seifert pairings of $W_{1}$ and $W_{2}$ are $R$-equivalent in $Z_{r, n}$. Since the homotopy Seifert pairing of $V_{\nu}$ is $R$-equivalent in $Z_{r, n}$ to that of $W_{\nu}, \nu=1,2$ (by Lemma 3.10(c)), Theorem 3.4 follows.

Theorems 3.3 and 3.4 give the main result of this paper:

3.14. THEOREM. Suppose that $r \geqslant 2, n \geq 4$. There is a mapping

$$
K_{r, n} \rightarrow Z_{r, n} / R
$$

which assigns to a knot the $R$-equivalence class of the homotopy Seifert pairing of any r-connected Seifert manifold of this knot. If $3 r \geqslant n+1 \geqslant 6$, then this mapping is bijective.

The first assertion of this theorem is true without assumptions that $r>2, n>4$ (see remark after the formulation of Theorem 3.4).

3.15. As an example we consider the class of simple odd-dimensional knots studied by $\mathrm{J}$. Levine and deduce from Theorem 3.14 an algebraic classification of such knots in terms of Seifert matrices. The result will be essentially the same as Levine's [10].

For this class of knots $n=2 q-1, r=q-1$. Suppose that $q>3$. Let $(K, \theta)$ be representative of some element from $Z_{q-1,2 q-1}$. This means that $K$ is a finite $(q-1)$-connected complex and $\theta: K \wedge K \rightarrow S^{2 q}$ is a homotopy pairing for which $\theta+\theta^{\prime}$ is a Spanier-Whitehead duality. It follows that $H^{i}(K ; G)$ is isomorphic to $H_{2 q-i}(K ; G)$ and therefore is equal to zero, for $i>q$. Thus $K$ has a homotopy type of bouquet of $q$-dimensional spheres. For each of these spheres choose a corresponding homology class $z_{i} \in H_{q} K$. Consider the integral square matrix $A$ with elements $a_{i j}=\theta_{*}\left(z_{i} \wedge z_{j}\right) \in H_{2 q}\left(S^{2 q}\right)=Z$. It follows from Hopf's theorem that two pairs $\left(K_{1}, \theta_{1}\right)$ and $\left(K_{2}, \theta_{2}\right)$ represent the same element of $Z_{q-1,2 q-1}$ if and only 
if the corresponding matrices $A_{1}$ and $A_{2}$ are congruent (this means that the integral unimodular square matrix $P$ exists such that $P^{\prime} A_{1} P=A_{2}$ ). Since $\theta+\theta^{\prime}$ is a Spanier-Whitehead duality, then $A+(-1)^{q} A^{\prime}$ is a unimodular matrix, where the prime means transposition. Thus the set $Z_{q-1,2 q-1}$ is in one-to-one correspondence with the set of congruence classes of integral square matrices $A$ for which $\operatorname{det}\left(A+(-1)^{q} A^{\prime}\right)= \pm 1$.

We shall call two integral square matrices $A_{1}$ and $A_{2}$, of sizes $r \times r$ and $s \times s$, respectively, contiguous if $r=s \bmod 2$ and an integral rectangular $(r \times s)$-matrix $B$ exists such that the matrix

$$
\left(\begin{array}{cc}
A & B \\
(-1)^{q+1} B^{\prime} & (-1)^{q+1} A_{2}^{\prime}
\end{array}\right)
$$

is congruent to matrix of the form $\left(\begin{array}{ll}0 & c \\ 0 & 0\end{array}\right)$, where $C$ is a square unimodular matrix of size $(r+s) / 2 \times(r+s) / 2$ and 0 means a zero square matrix of the same size (compare with Definition 2.3).

The equivalent definition may be obtained from the previous one replacing $C$ by the identity matrix.

We shall call two integral square matrices $A_{1}$ and $A_{2} R$-equivalent if there exists a sequence of integral square matrices $D_{1}, D_{2}, \ldots, D_{k}$ such that $D_{i}$ and $D_{i+1}$ are contiguous for $i=\overline{1, k+1}$, matrix $D_{i}+(-1)^{q} D_{i}^{\prime}$ is unimodular for $i=\overline{1, k}$ and $A_{1}=D_{1}, A_{2}=D_{k}$.

It is clear that the $R$-equivalence relation on $Z_{q-1,2 q-1}$ in the sense of 3.2 coincides, by means of the above-mentioned one-to-one correspondence, with the $R$-equivalence relation just introduced on the set of square matrices.

Now it follows from Theorem 1.14 that the map which assigns to a knot from $K_{q-1,2 q-1}$ the $R$-equivalence class of any Seifert matrix is a bijection of $K_{q-1,2 q-1}$ on the set of $R$-equivalence classes of square matrices $A$ with

$$
\operatorname{det}\left(A+(-1)^{q} A^{\prime}\right)= \pm 1 \text {. }
$$

It is not difficult to show that the $R$-equivalence relation on the set Seifert matrices coincides with the $S$-equivalence relation. It implicitly follows from the comparison of the results of the present paper with the work [10].

\section{REFERENCES}

1. G. E. Bredon, Regular $O(n)$ manifolds, suspension of knots, and knot periodicity, Bull. Amer. Math. Soc. 79 (1973), 87-91.

2. M. S. Farber, Classification of some knots of codimension two, Dokl. Akad. Nauk SSSR 240 (1978), 32-35; English transl., Soviet. Math. Dokl. 19 (1978).

3. J. F. P. Hudson, Embeddings of bounded manifolds, Proc. Cambridge Philos. Soc. 72 (1972), 11-20.

4. C. Kearton, Blanchfield duality and simple knots, Trans. Amer. Math. Soc. 202 (1975), 141-160.

5. __ An algebraic classification of some even-dimensional knots, Topology 15 (1976), 363-373.

6. R. Lashof and J. Shaneson, Classification of knots of codimension two, Bull. Amer. Math. Soc. 75 (1969), 171-175.

7. J. Levine, Unknotting spheres in codimension two, Topology 4 (1965), 9-16.

8. __ A classification of differentiable knots, Ann. of Math. (2) 82 (1965), 15-51.

9. ___ Polynomial invariants of knots of codimension two, Ann. of Math. (2) 84 (1966), 537-554.

10. An algebraic classification of some knots of codimension two, Comment. Math. Helv. 45 (1970), 185-198. 
11. C. P. Rourke, Embedded handle theory, concordance and isotopy, Topology of Manifolds, Markham, Chicago, Ill., 1970.

12. H. Seifert, Uber das Geschlecht von Knoten, Math. Ann. 110 (1934), 571-592.

13. E. H. Spanier, Function spaces and duality, Ann. of Math. (2) 70 (1959), 338-378.

14. Algebraic topology, McGraw-Hill, New York, 1966.

15. R. E. Stong, Notes on cobordism theory, Princeton Univ. Press, Princeton, N. J.; Univ. of Tokyo Press, Tokyo, 1968.

16. H. F. Trotter, On S-equivalence of Seifert matrices, Invent. Math. 20 (1973), 173-207.

17. C. T. C. Wall, Classification problems in differential topology-IV thickenings, Topology 5 (1966), 73-94.

18. G. W. Whitehead, Recent advances in homotopy theory, CBMS Regional Conf. Ser. in Math., No. 5, Amer. Math. Soc., Providence, R. I., 1970.

Institute of Cybernetics, Academy of Sciences, Azerbaijan S.S.R. 